\title{
Statistical analysis of solar proton events
}

\author{
V. Kurt ${ }^{1}$, A. Belov ${ }^{2}$, H. Mavromichalaki ${ }^{3}$, and M. Gerontidou ${ }^{3}$ \\ ${ }^{1}$ Institute of Nuclear Physics, Moscow State University, 119899 Vorobievy Gory, Moscow, Russia \\ ${ }^{2}$ Inst. of Terrestrial Magnetism, Ionosphere and Radio Wave Propagation, Russian Academy of Science (IZMIRAN), Russia \\ ${ }^{3}$ Nuclear and Particle Physics Section, Physics Department University of Athens, 15771 Athens, Greece
}

Received: 6 October 2003 - Revised: 11 February 2004 - Accepted: 8 March 2004 - Published: 14 June 2004

\begin{abstract}
A new catalogue of 253 solar proton events (SPEs) with energy $>10 \mathrm{MeV}$ and peak intensity $>10$ protons $/ \mathrm{cm}^{2}$.s.sr (pfu) at the Earth's orbit for three complete 11-year solar cycles (1970-2002) is given. A statistical analysis of this data set of SPEs and their associated flares that occurred during this time period is presented. It is outlined that 231 of these proton events are flare related and only 22 of them are not associated with Ha flares. It is also noteworthy that 42 of these events are registered as Ground Level Enhancements (GLEs) in neutron monitors. The longitudinal distribution of the associated flares shows that a great number of these events are connected with west flares. This analysis enables one to understand the long-term dependence of the SPEs and the related flare characteristics on the solar cycle which are useful for space weather prediction.
\end{abstract}

Key words. Interplanetary physics (Energetic particles; Flare and stream dynamics; Interplanetary shocks)

\section{Introduction}

Solar energetic particles, high-energy neutral emissions, coronal mass ejections (CMEs) and shock waves associated with fast CMEs determine the space weather at the Earth's orbit. The most powerful sources of solar energetic particle fluxes observed at $1 \mathrm{AU}$ are flares and interplanetary shock waves. The dynamic of energetic particles within the heliosphere involves the problems of acceleration, particles escaping and spreading near the Sun and propagation through the interplanetary medium. Actually, all of the above processes display a high degree of variability that results in the great diversity of the time behavior of the particle fluxes measured at 1 AU.

Significant progress has been made in the understanding of electromagnetic phenomena associated with the particle acceleration during the flares. The basic patterns of particle interplanetary transport and additional acceleration on

Correspondence to: H. Mavromichalaki

(emavromi@cc.uoa.gr) the interplanetary shock have been elaborated during the period between 1960 and 1980 (Dorman and Miroschnichenko 1968; Miroshnichenko, 2001). Pitch angle scattering plays an important role in the particle propagation. Transport includes the diffusion parallel and perpendicular to the mean magnetic field direction, focusing, drift motion under largescale field changes and sometimes "scatter-free" propagation. Acceleration can take place at a shock front with the help of additional scattering centers moving relative to the shock (McCracken et al., 1962; Roelof, 1969; Richter et al., 1981; Cliver et al., 1982; Valdes-Galicia et al., 1984; Mason et al., 1984; Forman et al., 1986; Reames D. V., 1999; Wibberenz et al., 1992).

For first time Van Hollebeke et al. (1975) using the data from the Goddard cosmic ray experiments on IMP-IV and $\mathrm{V}$, applied the procedure for identifying the associated flare of a solar proton enhancement and summarized the properties of 125 events in which the initiating flare location could be defined. The existence of a "preferable connection region" within $20^{\circ} \mathrm{W}$ to $80^{\circ} \mathrm{W}$ has been found. It was clarified that the maximum of the fluxes in each energy interval (energy spectrum) and high-energy threshold of the spectrum are the most important characteristics of solar energetic particle events. Cane et al. (1988) rested upon numerous original works and formulated that the intensity-time profiles of solar energetic particles display an organization with respect to heliolongitudes of parent flares, and the existing interplanetary shocks are the controlling agent. They explained the time behavior of solar energetic particles as a function of the longitude within the model framework for the large-scale structure of interplanetary shocks, which are probably driven by the piston of CME.

However, until now a persistent problem in the study of solar cosmic rays is the lack of exact information on the timing and on the conditions of accelerated protons spreading and escaping. One approach to understand the variability of the solar energetic particles fluxes measured at $1 \mathrm{AU}$ is a statistical study of their association with solar flares, with the shock wave and CME propagation on a large number of events. 

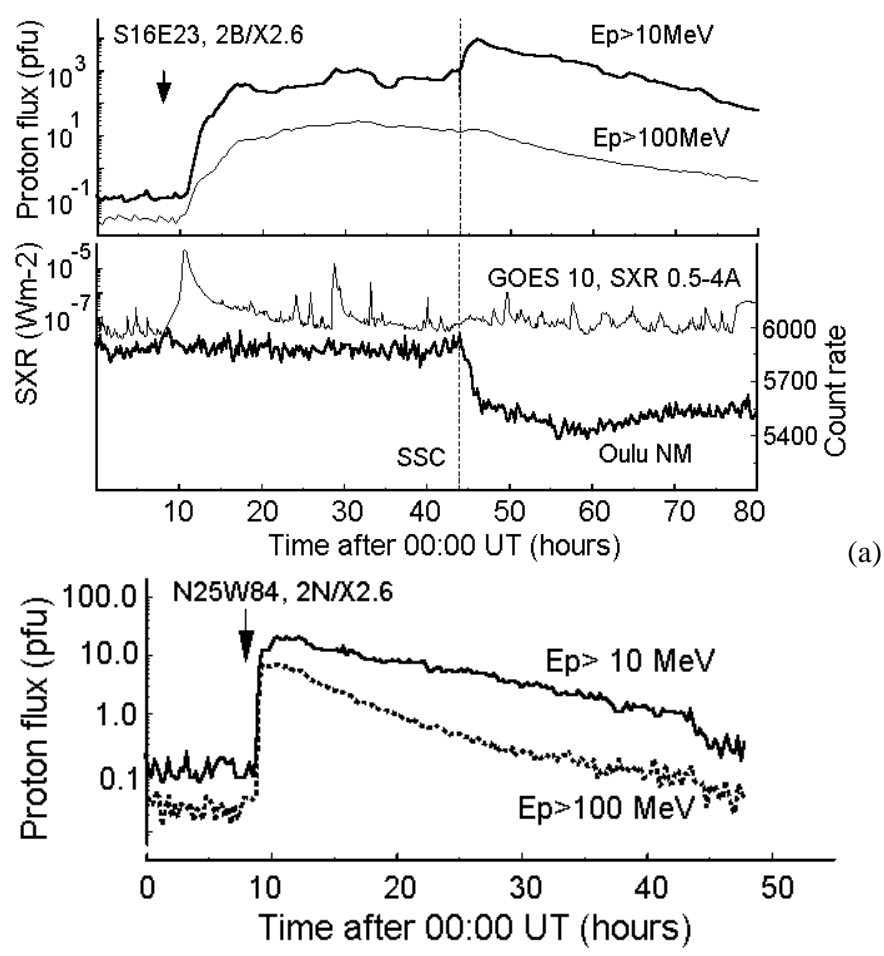

a)
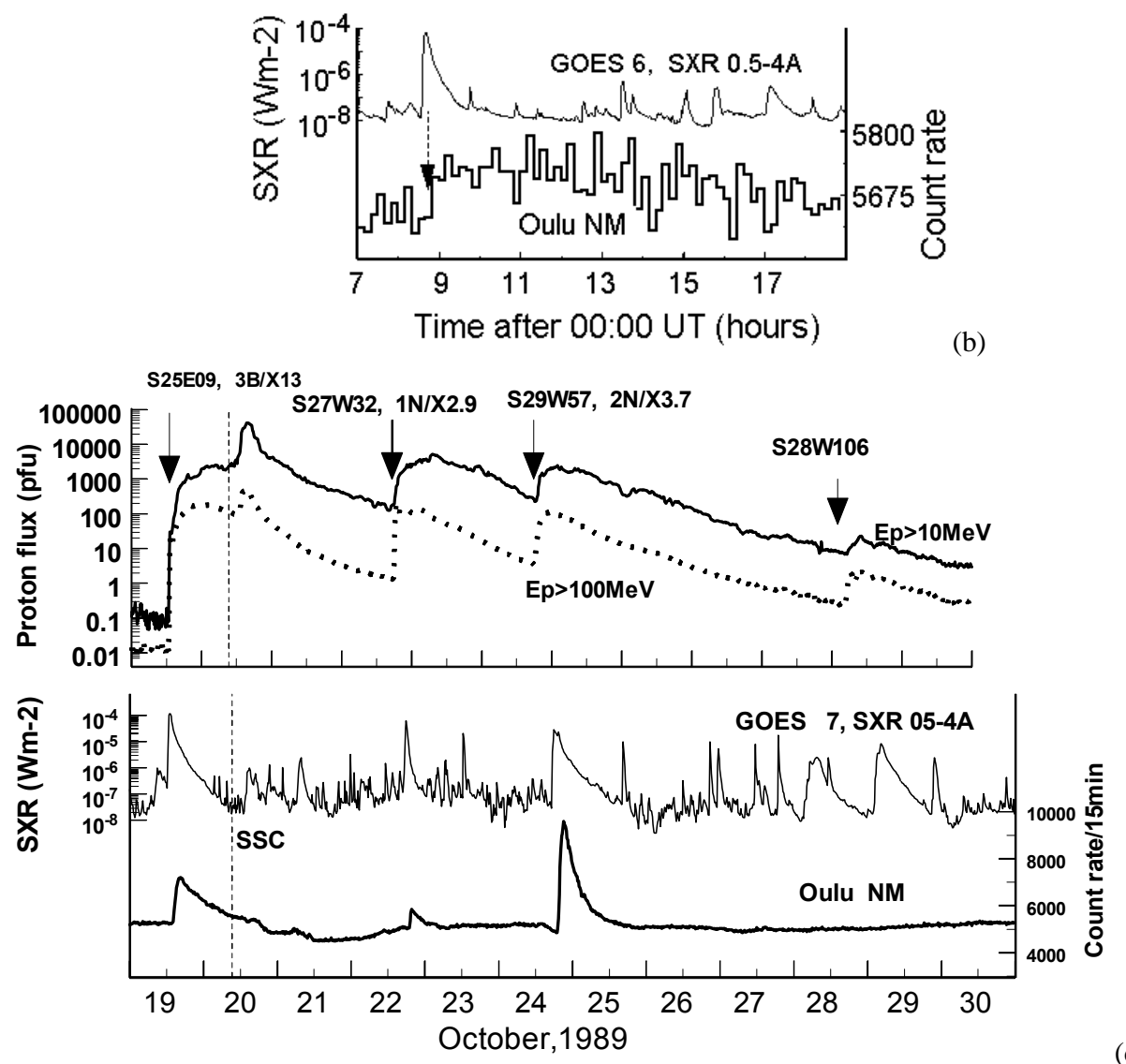

(c)

Fig. 1. Typical examples of large solar proton events which occurred on 24 September 2001, 25 July 1989 and during the time interval from 18 October to 31 October 1989 , respectively. 
According to the National Oceanic and Atmospheric Administration of the Solar Environment Center (NOAA/SEC) definition, a "solar proton event" (SPE) is the solar energetic particles' enhancement in which proton flux with energy $\mathrm{Ep}>10 \mathrm{MeV}$ is greater or equal to $10 \mathrm{part} / \mathrm{cm}^{2} . \mathrm{s} . \mathrm{sr}$ (10 pfu) up to the background level near $1 \mathrm{AU}$. The onset time of a proton event is defined by the first three consecutive $5 \mathrm{~min}$ average data points with fluxes greater than or equal to $10 \mathrm{pfu}$. The end of the event is the last time when the flux was greater than or equal to $10 \mathrm{pfu}$. This definition allows for multiple proton enhancements to be considered as one proton event.

Several catalogues of SPEs have been created and their data are analyzed within the framework of particle acceleration in different sources at or near the Sun (Van Hollebeke et al., 1974; Svestka and Simon, 1975; Basilevskaya et al., 1983; 1986; Sladkova et al., 1990; 1998; Cliver et al., 1991; Stolpovsky et al., 1988; Goswami et al., 1988; Shea and Smart, 1990; Feynmann et al., 1993; Gabriel and Feynmann, 1996; King 1984; Crosby et al., 1993; Mendoza et al., 1997; Gerontidou et al., 2002)

This work presents a new updated catalogue of solar energetic particles events for the time interval 1970-2002. Statistical properties of SPEs and their association with neutron monitor enhancements as well as some properties of the solar flares, identified as "associated", are discussed. This catalogue is mainly based on the catalogue of solar proton events edited by Logachev at the Institute of Nuclear Physics of Moscow University (Basilevskaya et al., 1983; 1986; Sladkova et al., 1990; 1998) that covers the time span 1970-1996. In these issues the particle flux time profiles are taken from the Meteor satellite observations (Fedorov, Institute of Applied Geophysics), GOES, IMP and balloon (Lebedev Physical Institute of Russian Academy of Sciences) measurements and Neutron Monitor Network (NMN), as well. The intensity-time profiles of proton fluxes in several energy bands, the integral proton energy spectrum, as well as information on the possible SPE sources for each event, can be found in these issues. Listing of these events was extended up to now using the issues from NOAA SEC (2002). The NMN database of IZMIRAN (http://www.izmiran.rssi.ru) was also used for identification of the ground level enhancements (GLEs).

\section{Catalogue description}

Protons can be accelerated to energies more than $10 \mathrm{MeV}$ during the flare development or near the shock front associated with the CME propagation. Thus, when one speaks about the source of the solar proton event, these two different sources are mentioned. Time profiles of SPEs appear to be different for these two sources and depend on the relative position of the observation point.

In order to demonstrate this difference among SPE characteristics, time profiles for three different cases of SPEs are presented in Fig. 1a, 1b and 1c. A typical solar proton event which occurred on 24 September 2001 is given in Fig. 1a.
The "associated flare" of this event occurred with importance 2B/X2 and was located at S16 E23, while the soft X-rays duration was about 97 minutes. A coronal mass ejection and a shock wave were associated with this flare. When the interplanetary disturbance reached the Earth, a strong geomagnetic storm and a big Forbush decrease started on 25 September at 20:25 UT. The peak values of $10 \mathrm{MeV}$ proton flux was achieved at about 08:00-09:00 UT on 26 September, simultaneously with the minimum value of cosmic ray intensity recorded at NM stations. Time profile of the SPE exhibits two strongly pronounced increases; nevertheless, according to the NOAA SEC definition, proton enhancement is considered as "one" event, and maximum flux values (Tmax and Imax) have been attributed to fluxes registered simultaneously with the shock.

An exceptional event of our catalogue is the event of 25 July 1989, presented in Fig. 1b. In spite of the fact that the peak flux for $\mathrm{Ep}>10 \mathrm{MeV}$ protons was only three times greater than the threshold intensity (10 pfu), it was recorded as ground level enhancement at the Earth. The time profile of $10 \mathrm{MeV}$ protons was very sharp with a time rise of about 180 minutes. An associated flare with importance $\mathrm{X} 2.6 / 2 \mathrm{~N}$ was located at $\mathrm{N} 25 \mathrm{~W} 84$ and had a total duration of 29 minutes.

The widely known time interval from 18 October to 31 October 1989 is the period with the highest proton fluxes over the history of space explorations. The spectacular time profiles of the protons are presented in Fig. 1c. A powerful flare at 19 October with importance 3B/X13 was the first one in the series of long duration SXR flares accompanied by $\mathrm{SPE}$. The maximum flux was registered simultaneously with sudden storm commencement (SSC) and Forbush decrease at 09:20 UT on 20 October. Three GLEs were recorded at neutron monitors on 19, 22 and 24 October. The GLEs on 19 and 24 October, having at the South Pole neutron monitor magnitudes greater than of $90 \%$ and $200 \%$, respectively, are among the biggest GLEs of the solar cycles 20-23.

In this study the associated sources of each solar proton event with peak flux $>10 \mathrm{pfu}$ were found. Contrary to the NOAA/SEC definition, we tried to find and to distinguish a diffusion maximum of each event. Of course, there are events without any association with flares and when an SPE is created by far eastern flares, the associated flare identification is poor. The time profile of these events exhibits only one maximum that coincided in time with the sudden commencement occurence. In this case this is adopted as the maximum value of the event. A list of 253 solar proton events with energy $>10 \mathrm{MeV}$ and peak intensity $>10$ pfu over the time period 1970-2002 is presented in Table 1. After the numbering of the events the first two columns of this Table are the date and the onset time of each event. The time interval with intensity $\geq 90 \%$ of the peak flux (peak duration) and peak flux are given in the next two columns. 


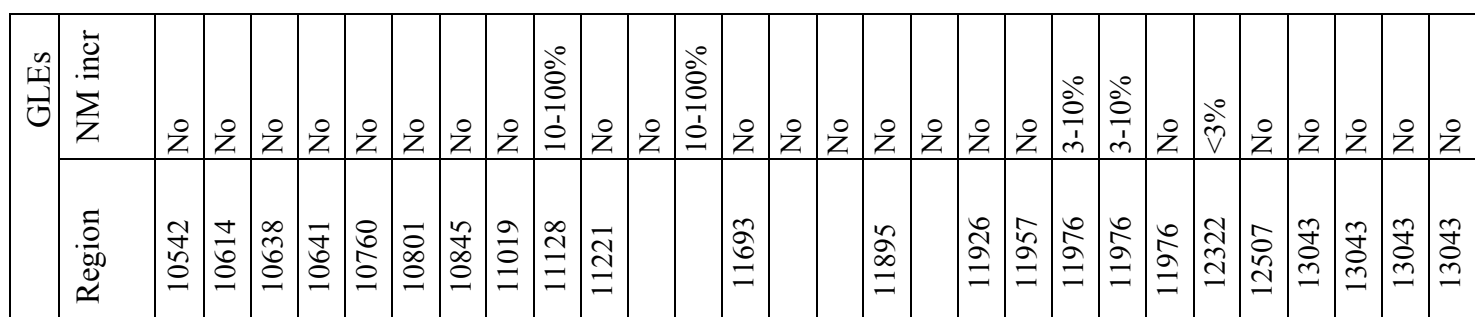

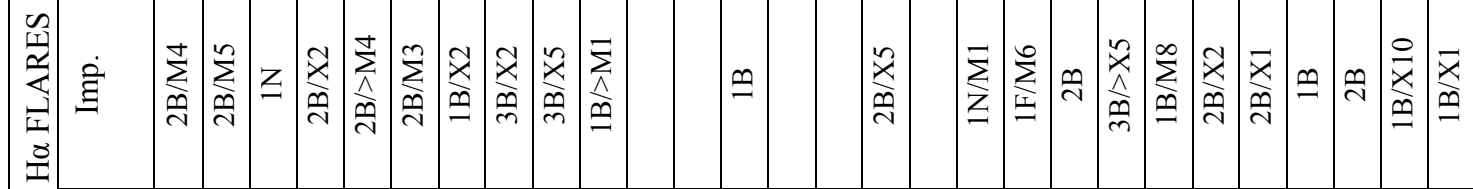

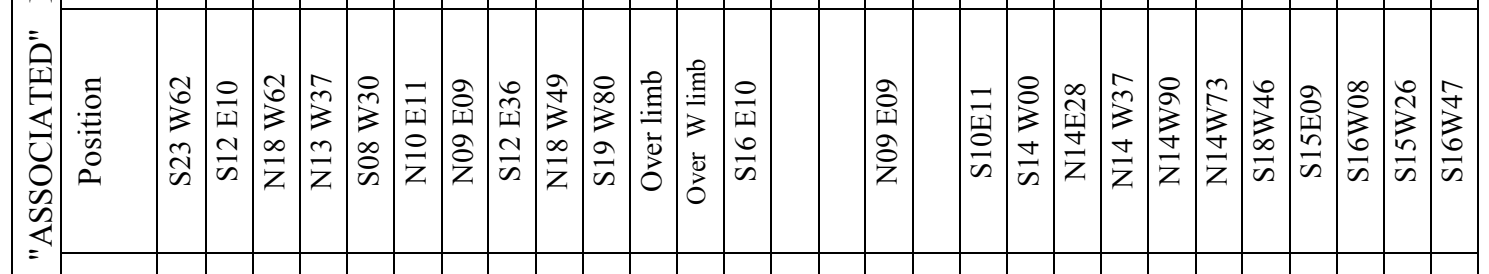

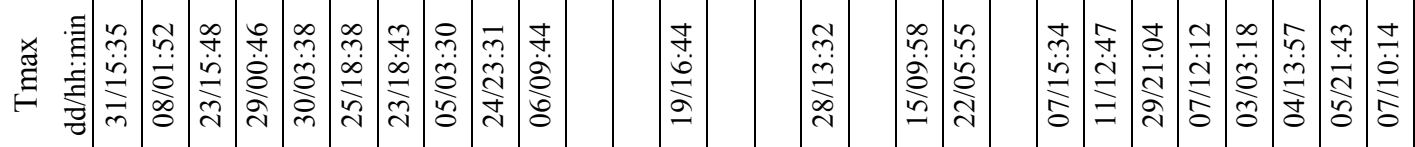

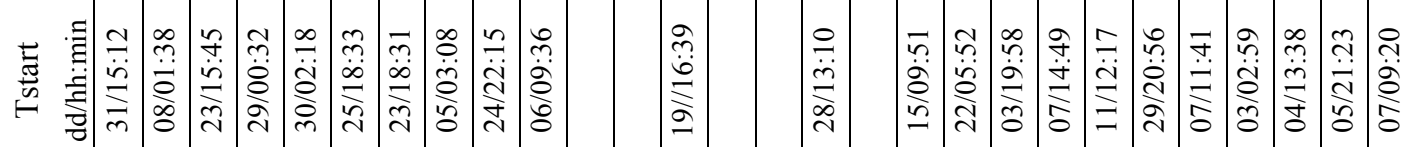

着 离

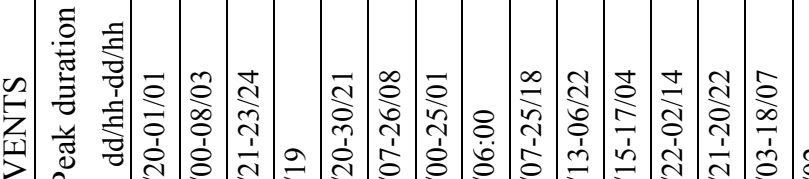

t)

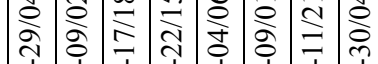

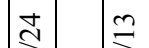

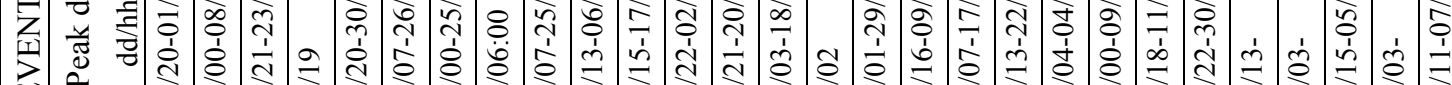

$\wedge$

离

売

孞

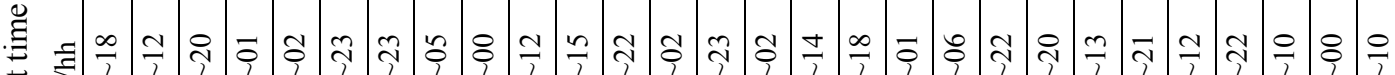

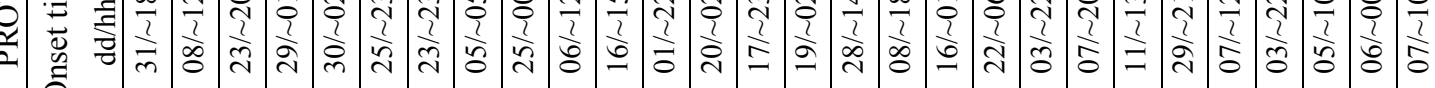

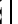

o

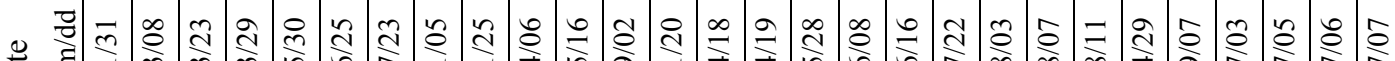

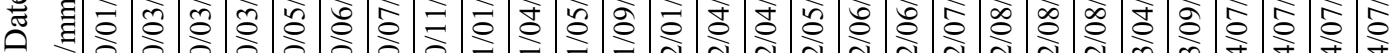

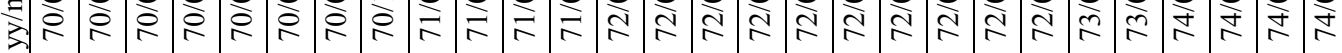

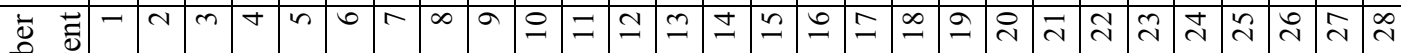
吾至 
z z z z z

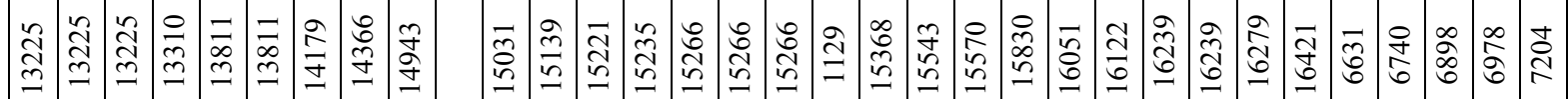

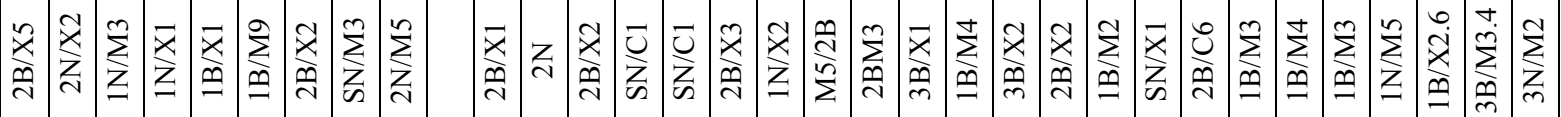

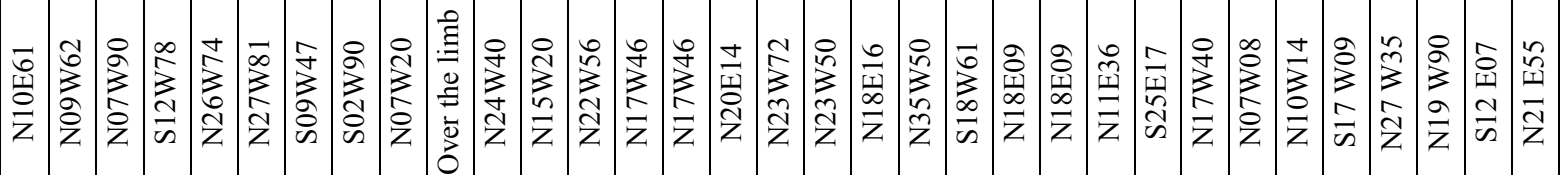

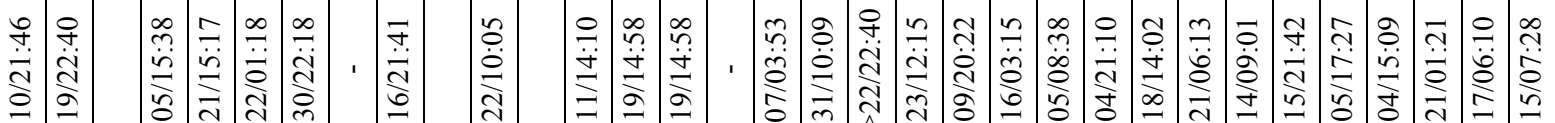

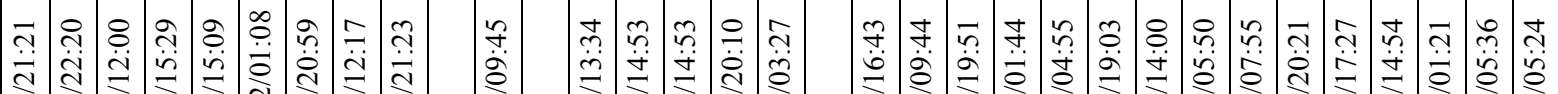

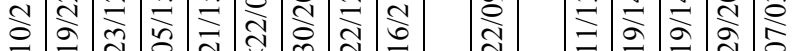
तิ

\&

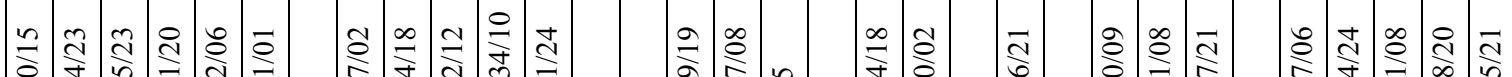

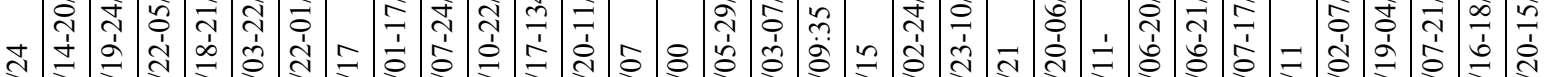

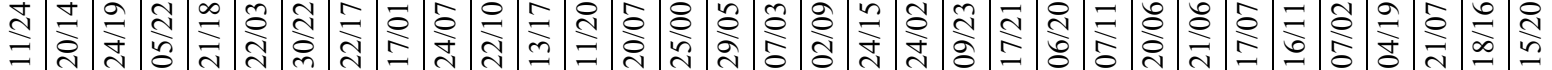

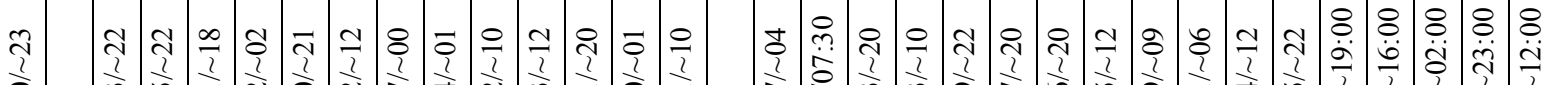

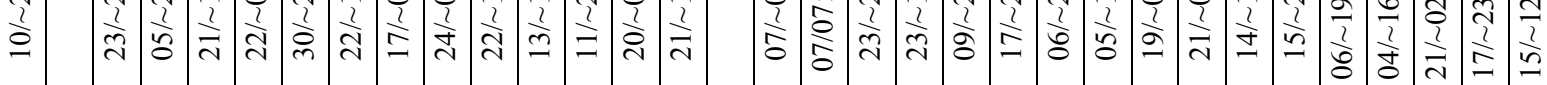

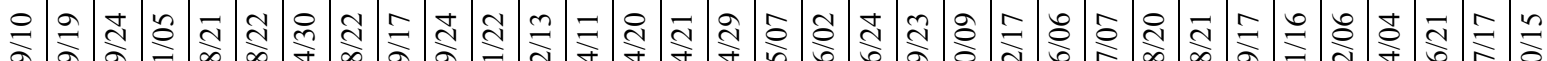
ஓे

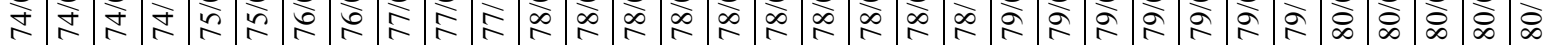

ते 


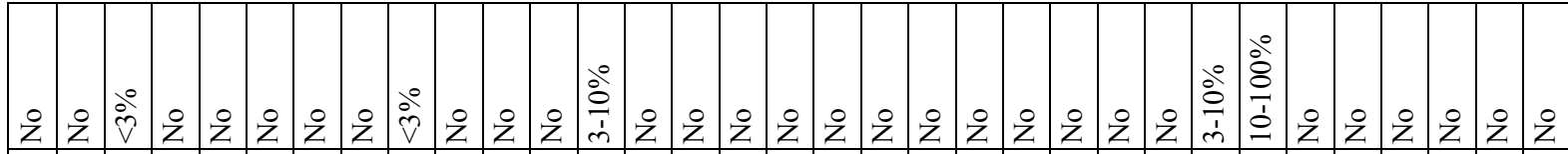

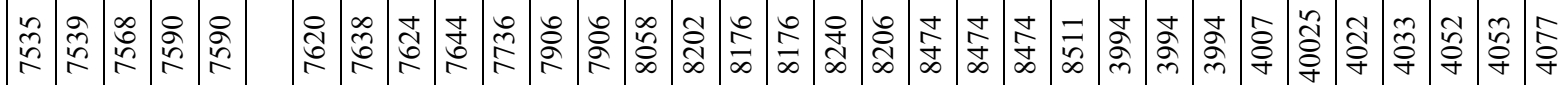

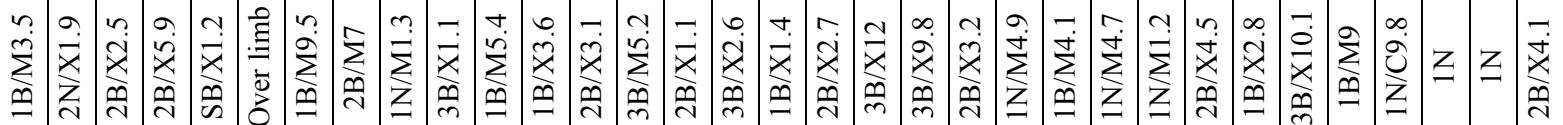

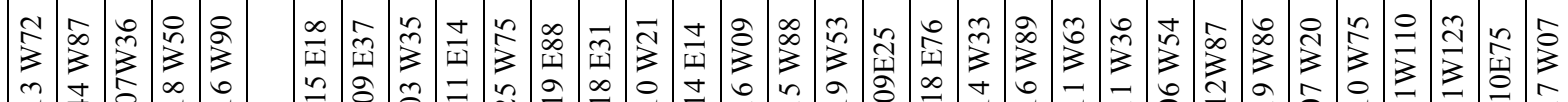

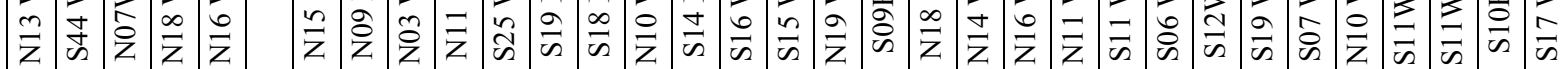

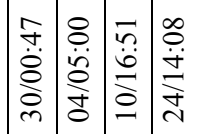

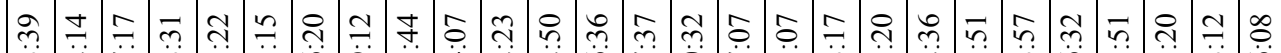

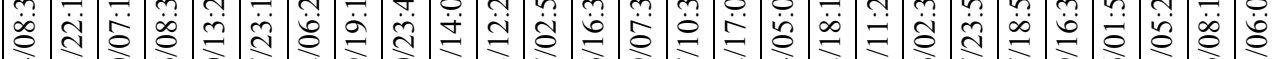

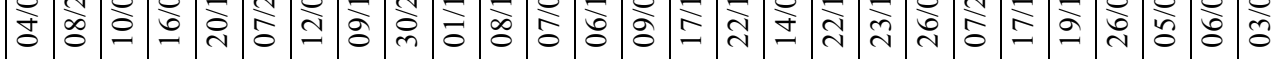

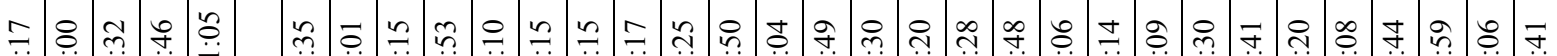

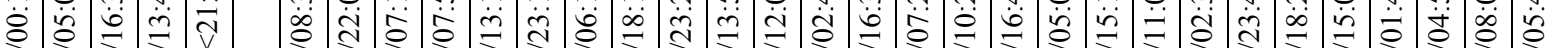

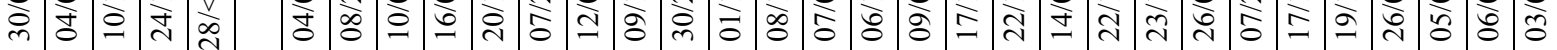

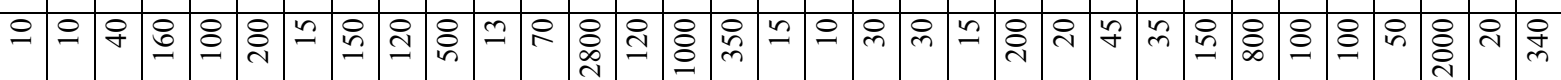

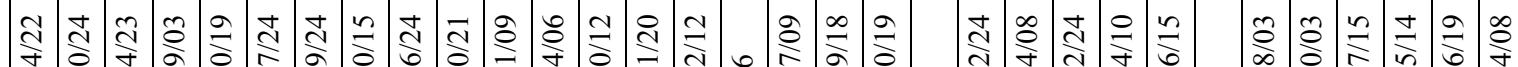

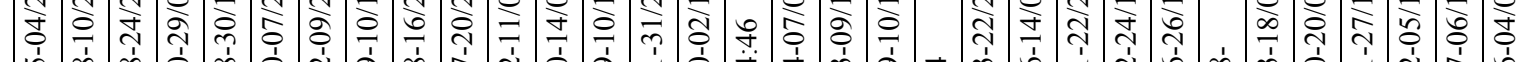

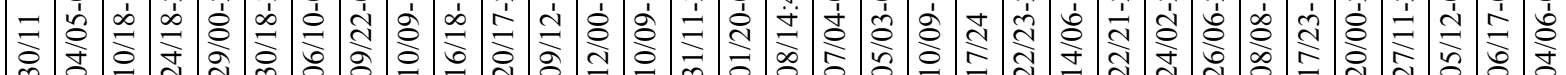

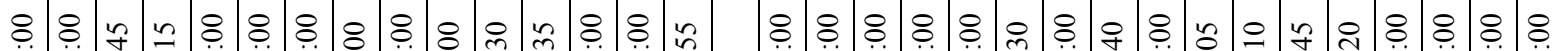

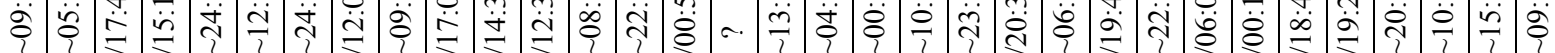

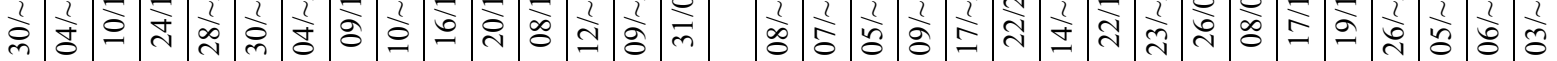

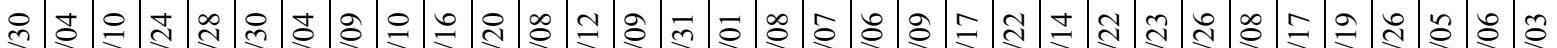

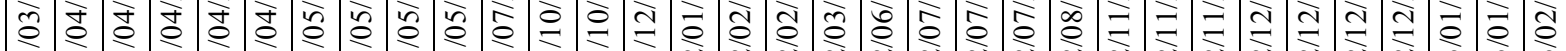

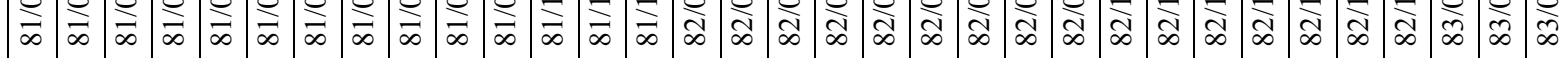
둥 


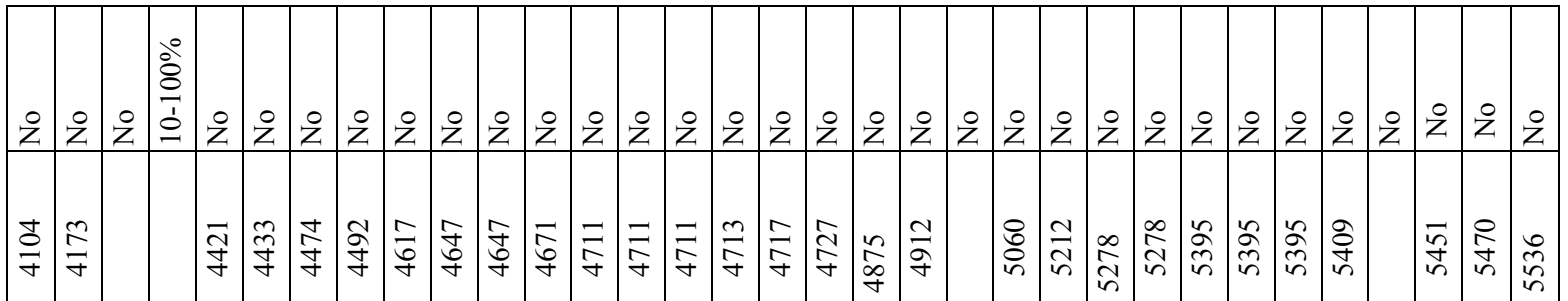

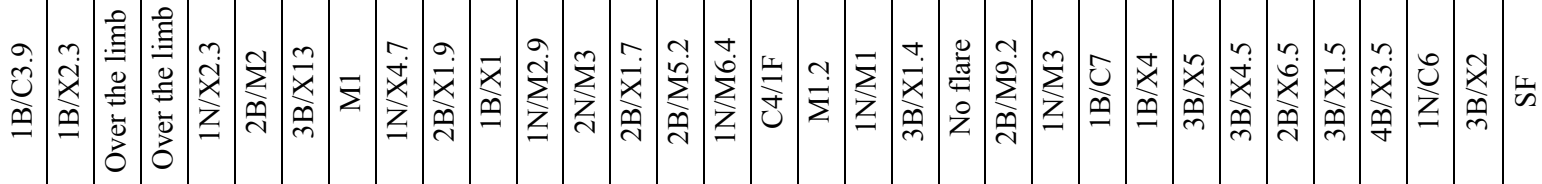

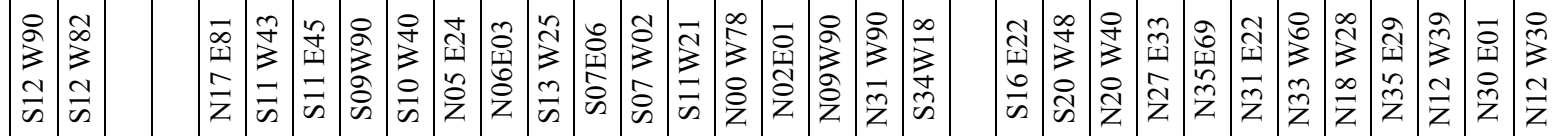

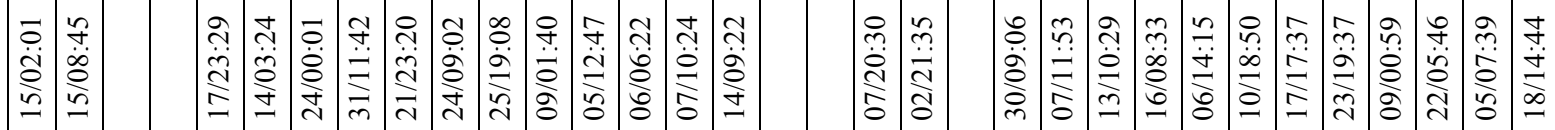

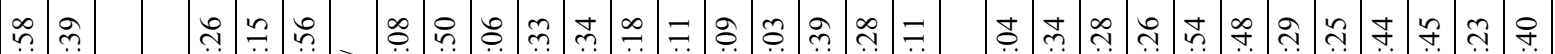

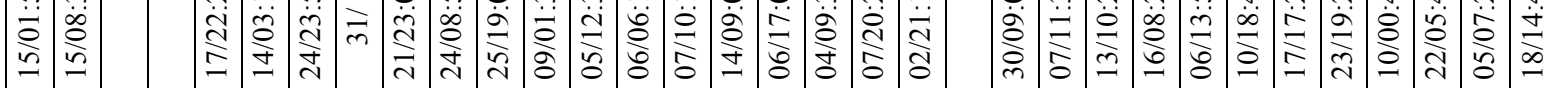
$\therefore$ 으의

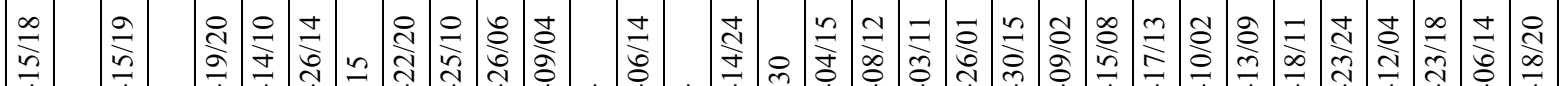

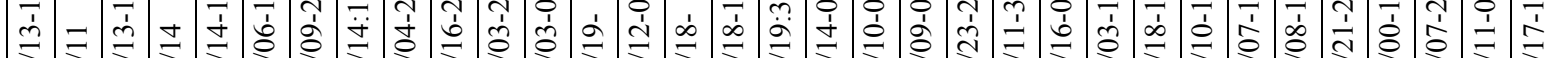

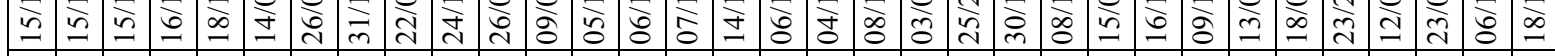
8 8 .

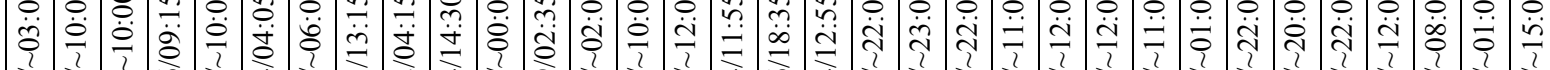

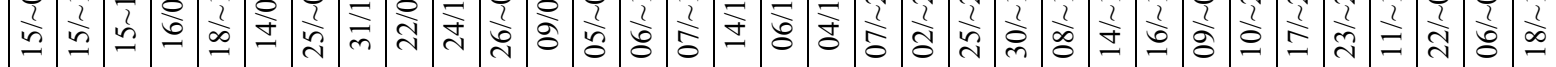

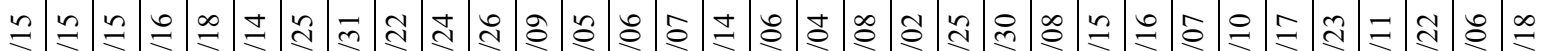

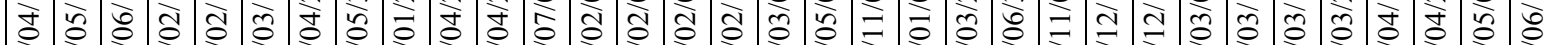

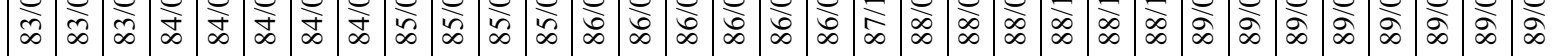

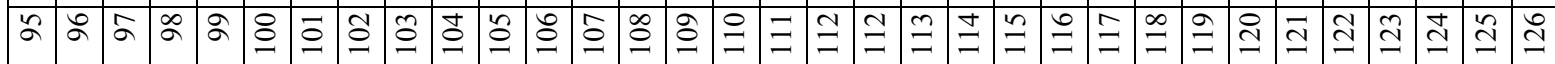




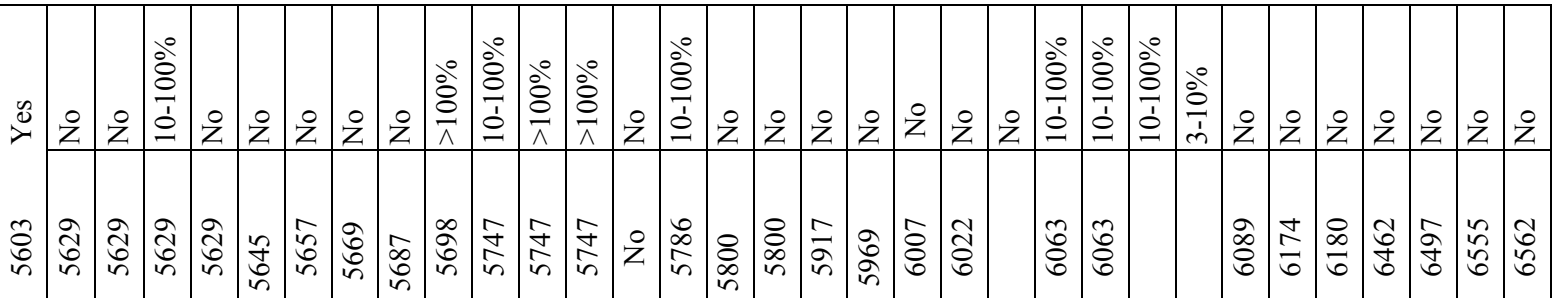

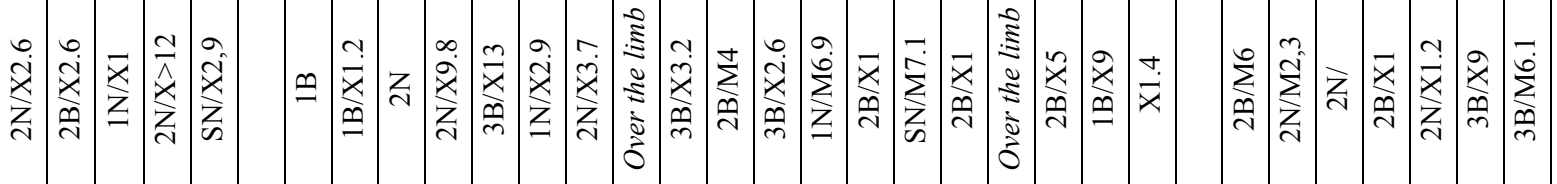

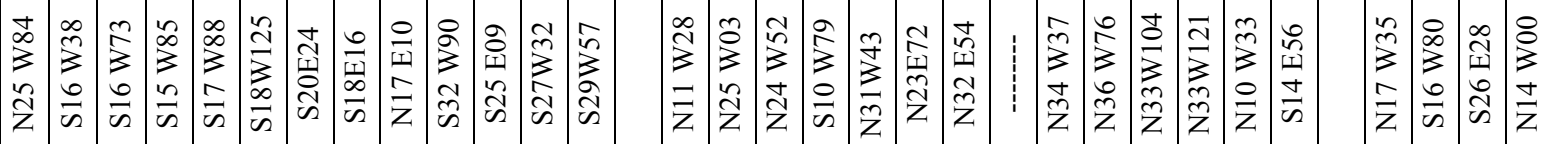

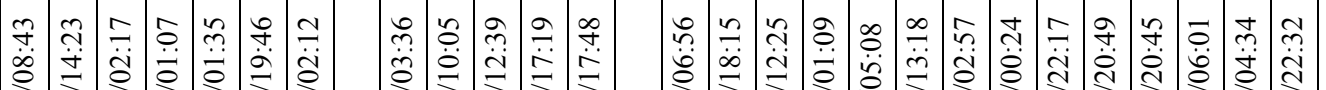

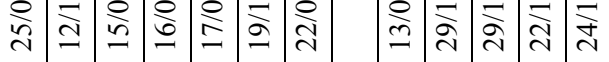
方 8 ㄴำ

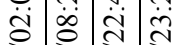
ले भूत

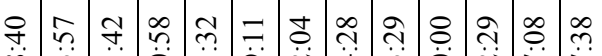

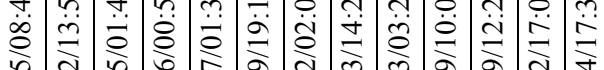

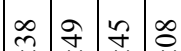

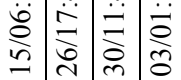

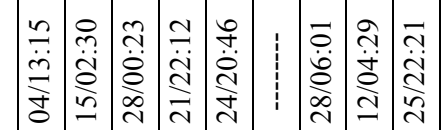
in গิ

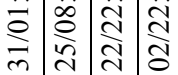

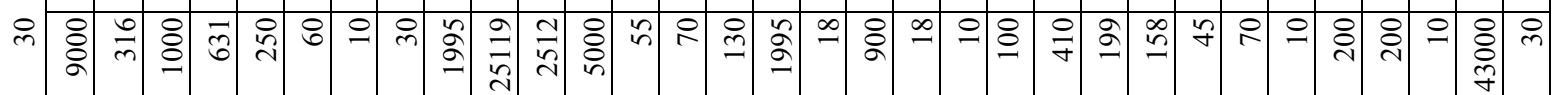

J

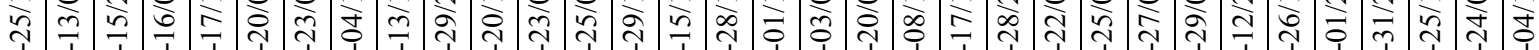

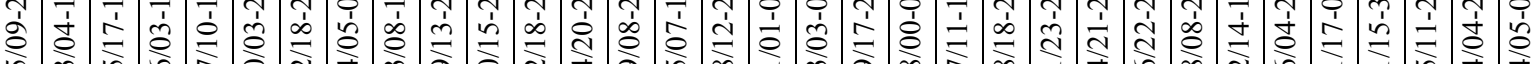

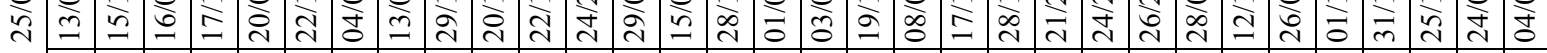

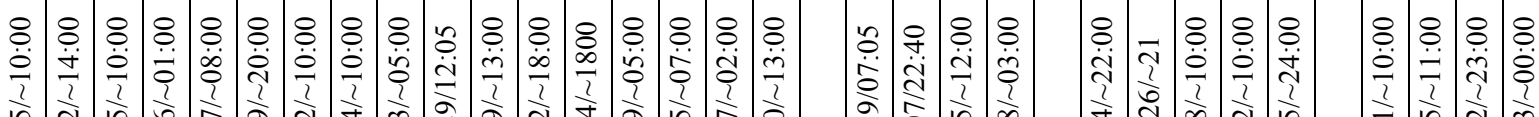

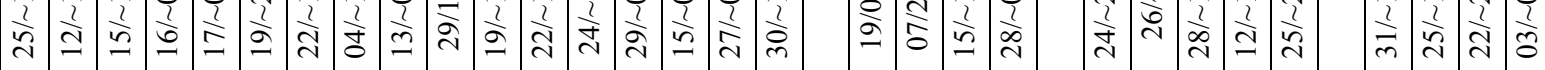

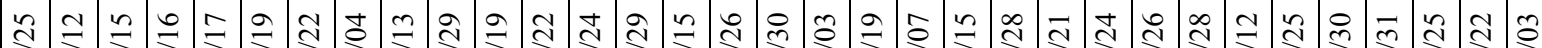
它 ळे

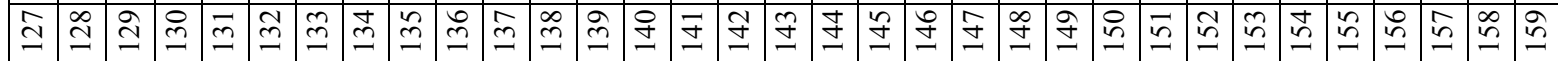




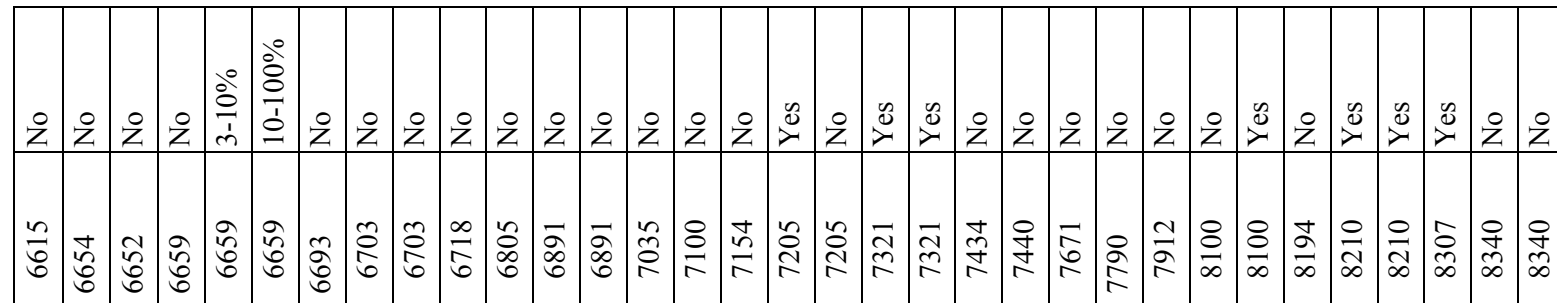

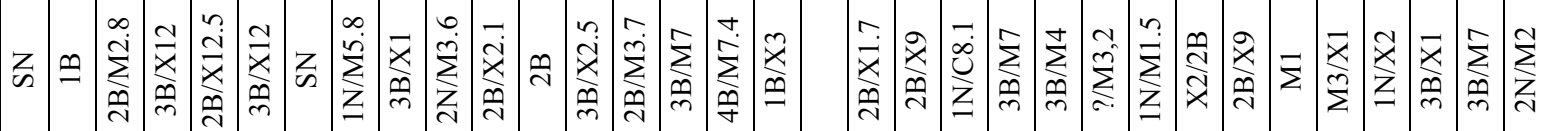

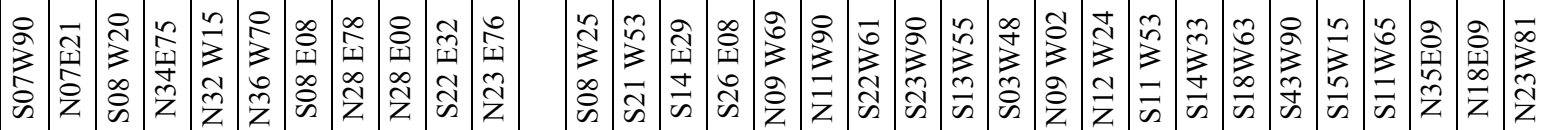

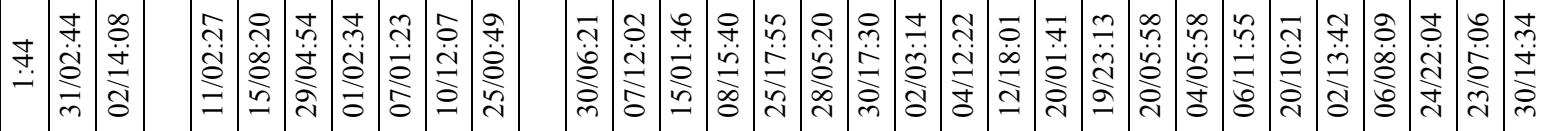

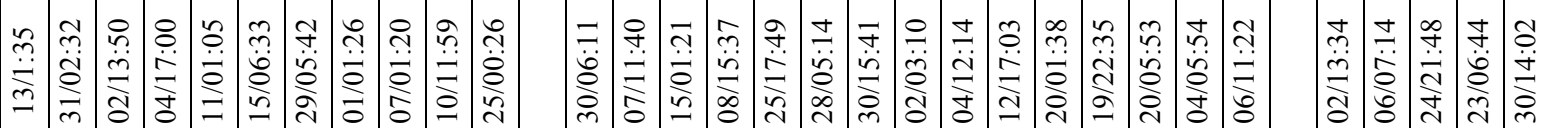
\&

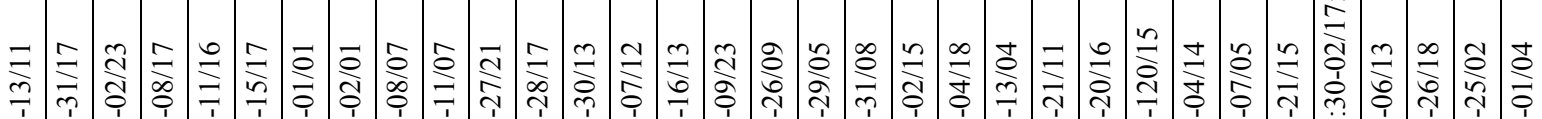

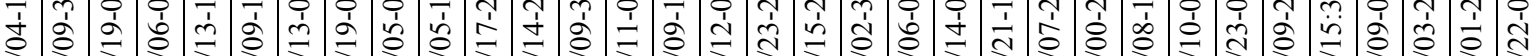

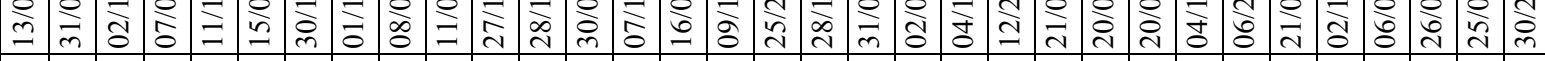
\&

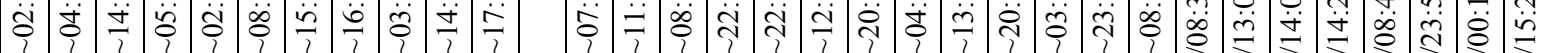

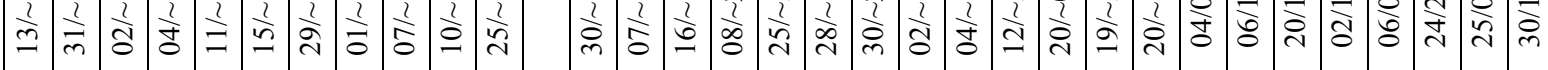

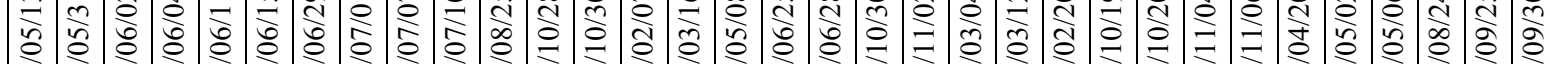
৯

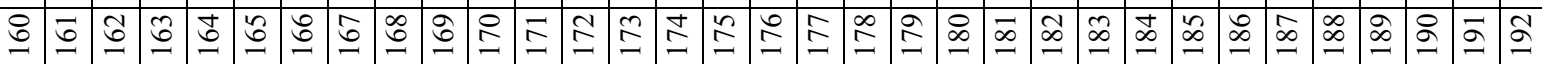


ż $そ$ ż

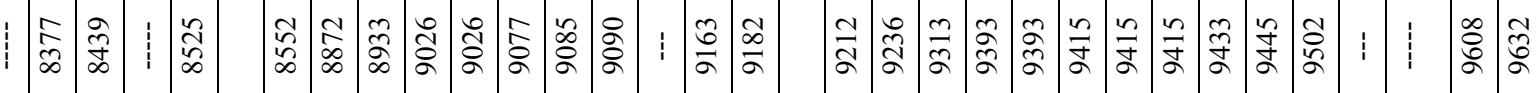

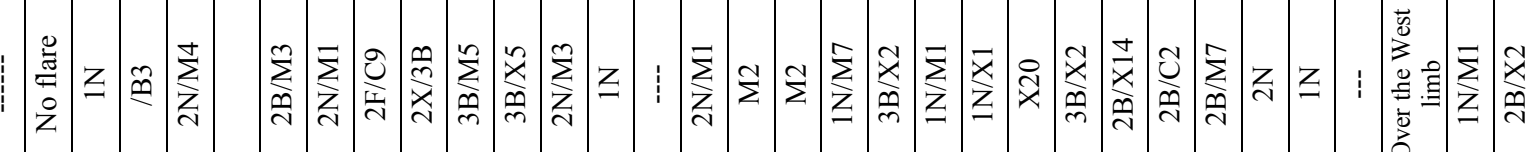

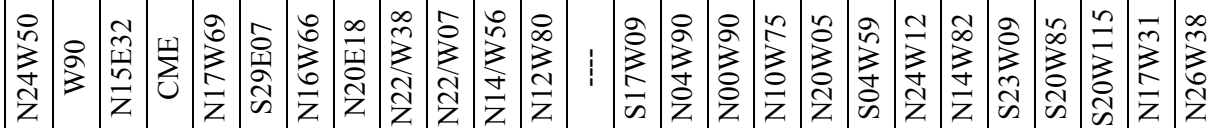

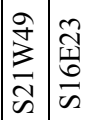

m

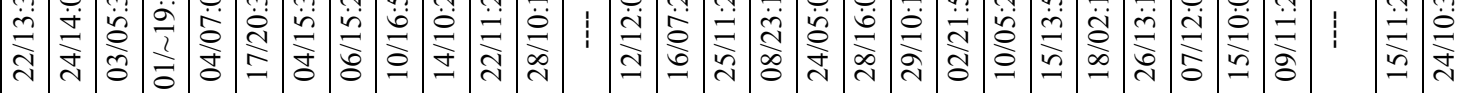

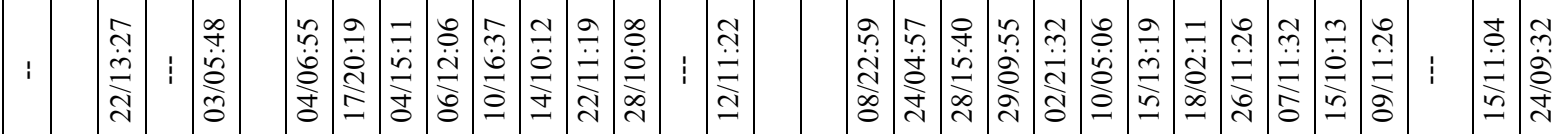

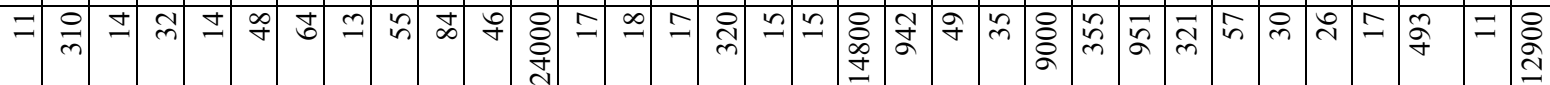

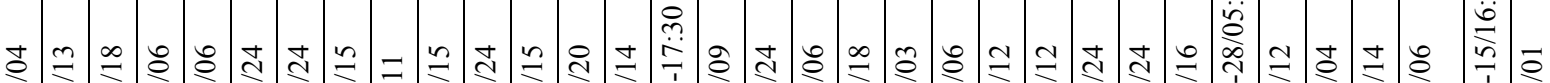

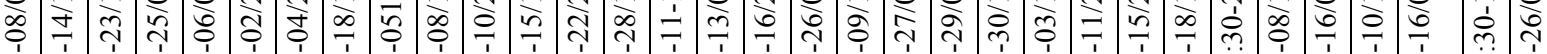

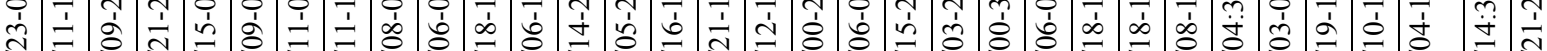

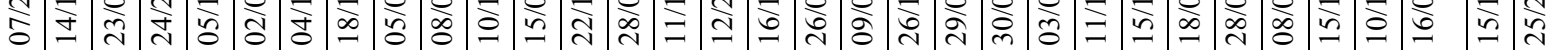
ㄴ

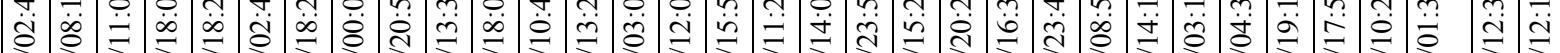

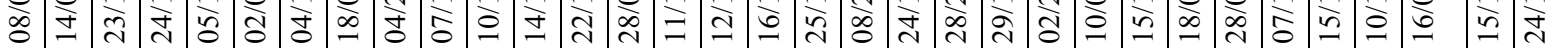

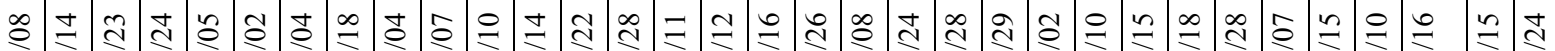

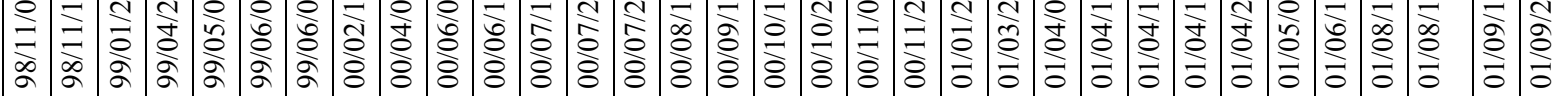

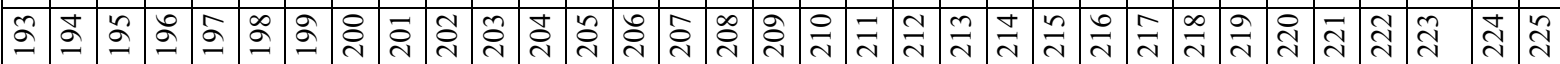




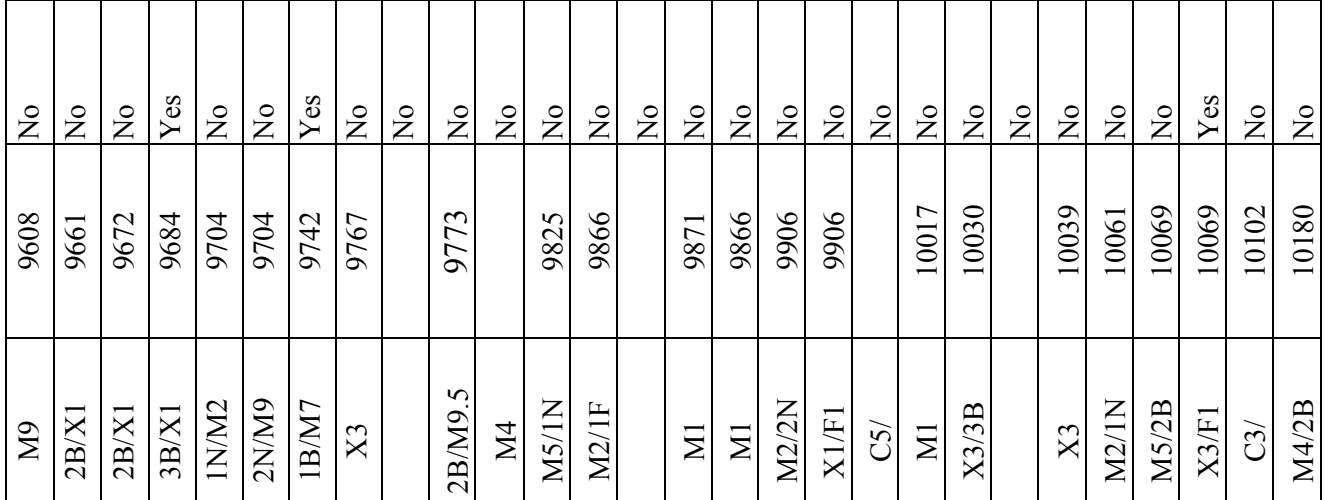

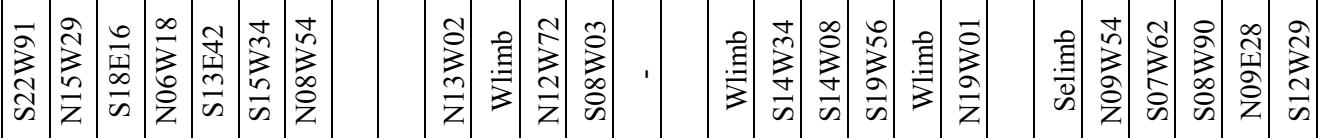

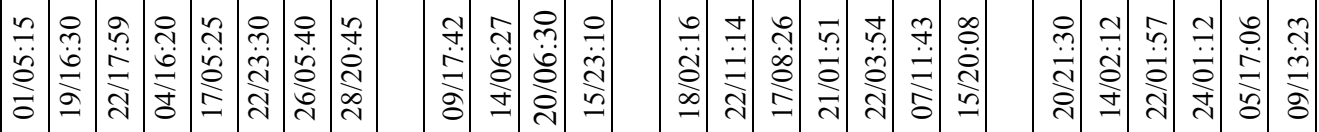

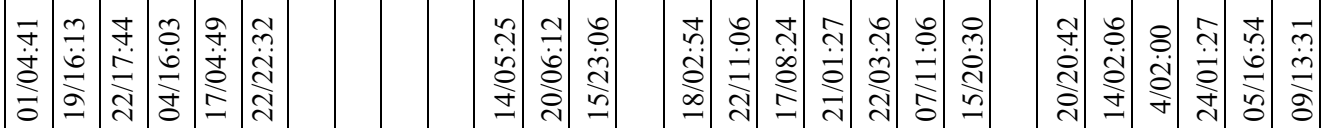

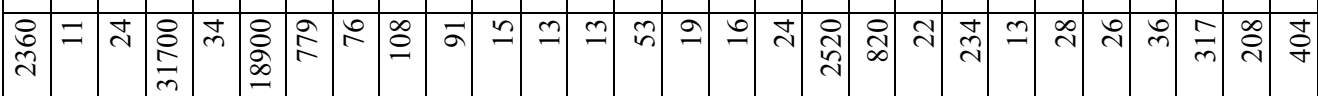

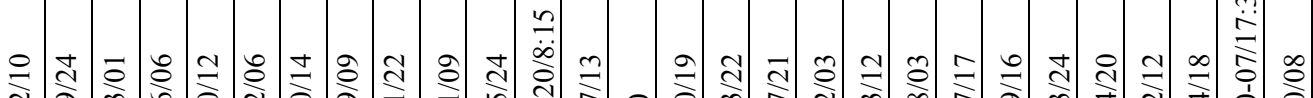

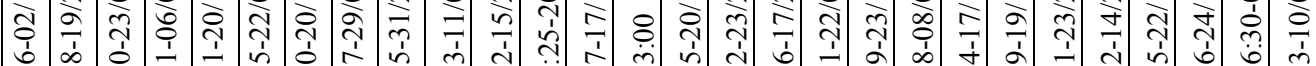

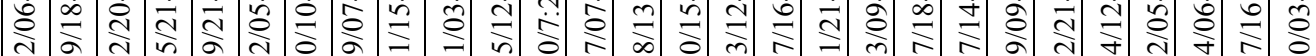

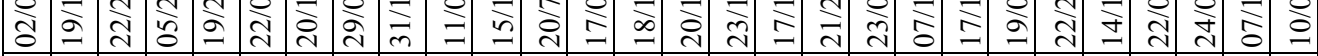

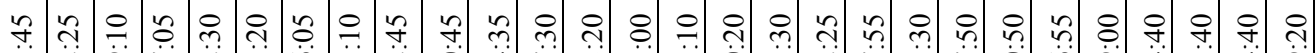

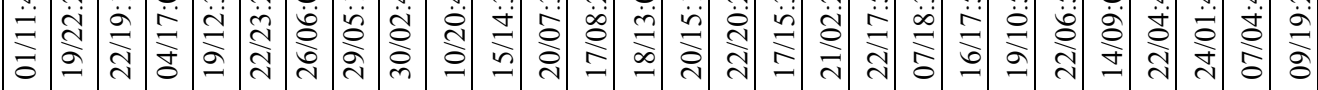

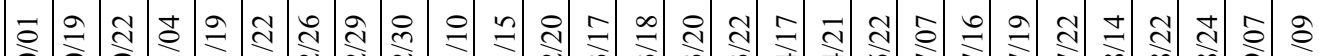

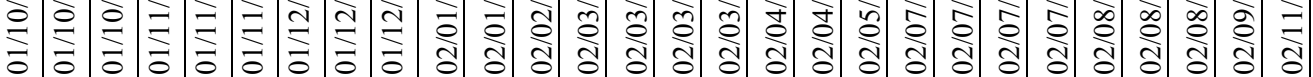

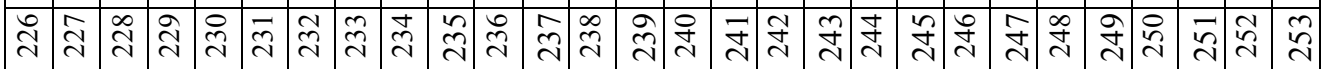



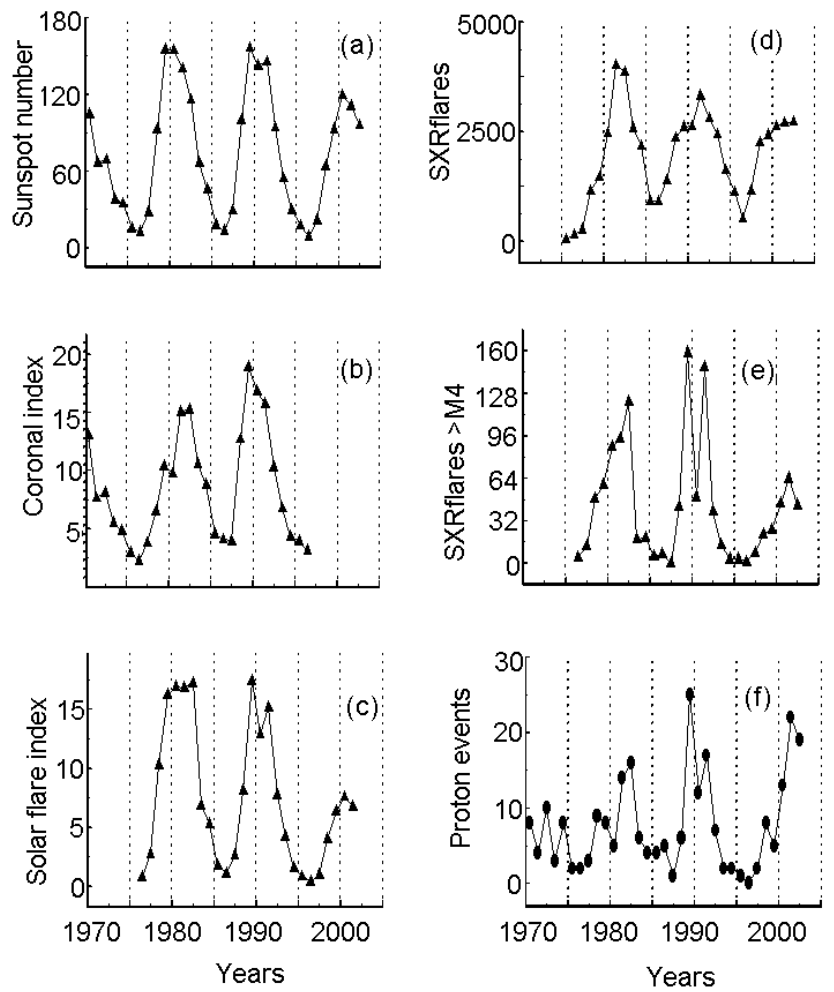

Fig. 2. Time distributions of the yearly values of (a) sunspot number, (b) coronal index, (c) solar flare index, (d) SXR flares number, (e) SXR flares number with importance $>$ M4 and (f) proton event number with $\mathrm{E}>10 \mathrm{MeV}$ and peak intensity $>10 \mathrm{pfu}$, during the time interval 1970-2002.

The next five columns concern Ha flares identified as the source of SPEs. These columns consist of onset time, peak time, importance, as well as SXR importance (by GOES classification), flare location and active region number. The last column of the Table indicates cosmic ray variations recorded at the neutron monitors as ground level enhancements. With this catalogue we obtain a possibly homogeneous data set comprising 253 SPEs that provides a significant statistical basis. Furthermore, as the catalogue covers the descending phase of the cycle 20, two solar cycles, 21 and 22, and the ascending phase of the cycle 23 , it enables us to analyze the long time dependency of the SPEs and flare-related characteristics (Temmer et al., 2001).

In our Table only large events with energy $>10 \mathrm{MeV}$ and peak intensity $>10$ pfu are included, while smaller events with intensity $<10$ pfu are not selected (Shea and Smart, 1990). Possible differences between our Table and other relative tables are due to the fact that this Table is mainly based on the catalogue of solar proton events edited by Logachev (Moscow University) which is not commonly used. This catalogue is a very useful issue, as it collects measurements from Neutron Monitors, balloons and different satellites containing time profiles and combined spectra of each event with maximum intensity $>1$ pfu.

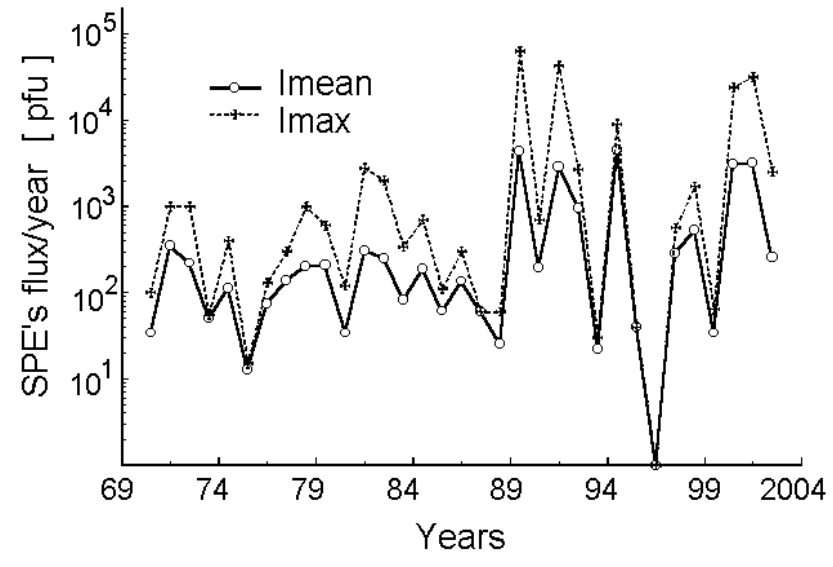

Fig. 3. Time distribution of yearly averaged and maximum intensity of the SPEs flux from 1970 to 2002.

\section{Statistical treatment}

\subsection{Correlation analysis}

The relationship of SPEs number with different manifestations of solar activity, such as sunspot number Rz, coronal index (total irradiance of the green corona in line $5303 \mathrm{~nm}$; Rusin and Rybansky, 2002), solar flare index (total energy emitted by $\mathrm{H}_{\alpha}$ flares; Ozguc and Atac, 1994), SXR flares registered by GOES satellites, is analyzed. All relevant data are extracted from NOAA SEC data base.

Time distributions of these solar activity indices, as well as of the number of SXR flares of importance $>$ M4 over the time interval 1970-2002, covering more than three solar activity cycles, are presented in Fig. 2. We can say that sunspot numbers, coronal index, solar flare index and SXR flare number differ by their maximum values in cycles 21 and 22 by not more than $20 \%$. On the contrary, the number of solar proton events in the maximum of cycle 22 is double in comparison with cycle 21 . The events distribution for solar cycle 22 differs completely from those in the previous cycle. About $70 \%$ of these events originated during the three years at the maximum of this cycle, that is in agreement with the result of Shea and Smart (1995). The results can also be confirmed by the calculation of maximum and yearly averaged intensity of the SPEs flux. These intensities appear to be ten times smaller in the period 1970 to 1988 than those in interval 1989 up to 2002, as can be seen in Fig. 3. The increased occurrence rate of the SPEs during solar cycle 22 is in accordance with the number of solar flares of $\geq \mathrm{M} 4$ importance.

Comparing yearly values of proton events with sunspot number, coronal index, solar flare index, total SXR flares and SXR flares of $\geq \mathrm{M} 4$ importance, we have found that the largest values of the correlation coefficient among these parameters are those for coronal index $(0.86 \pm 0.05)$ and for SXR flares with importance $\geq M 4(0.81 \pm 0.07)$. Scatter plots and linear approximations of the SPE yearly number dependences on the above mentioned parameters, are given in Fig. 4. We do not discuss how the coronal index is connected 

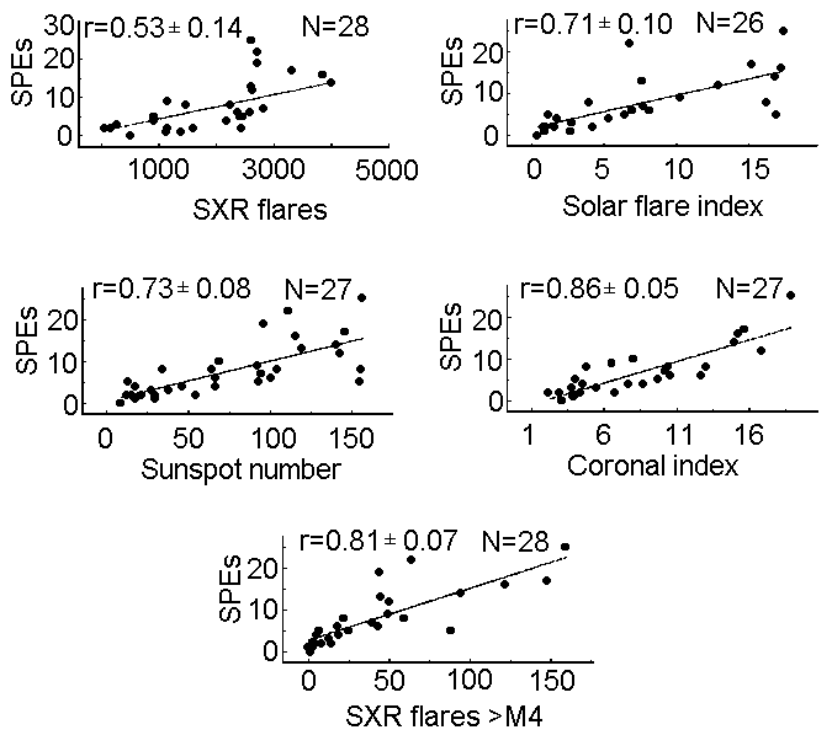

Fig. 4. Scatter plots and linear approximations of SPEs yearly number dependences on the total SXR flares, sunspot number, solar flare index, coronal index and SXR flares with importance $>$ M4 .

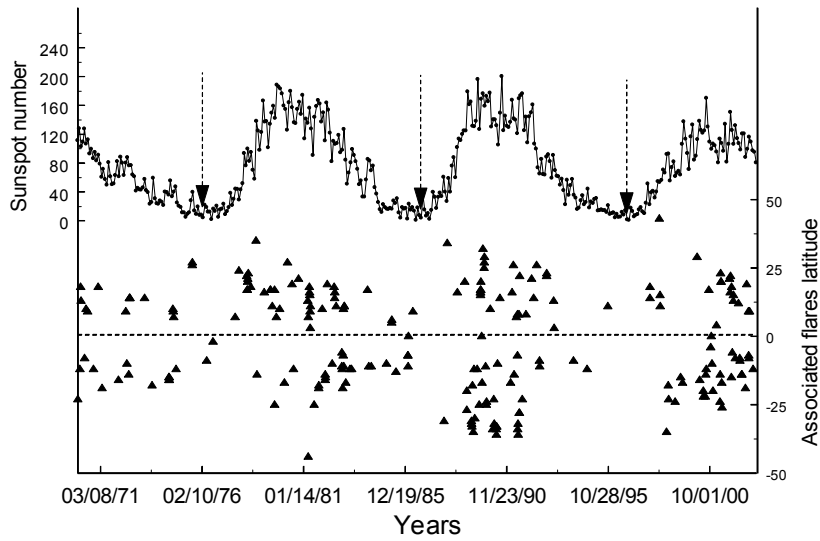

Fig. 5. "Butterfly diagram" of the associated with SPEs flares for the interval 1970-2002 is presented in the lower curve. Time profile of the sunspot number is also given in the upper curve.

with the high-energy particle production, but this fact is remarkable. From the other side it is natural to assume that solar activity with soft $\mathrm{X}$-rays of importance $\geq \mathrm{M} 4$ have an ability to emit into interplanetary space protons sufficient to be registered near the Earth's vicinity as SPEs (Kurt, 1990; Belov et al., 2001).

The interplanetary magnetic field lines constitute an Archimedian spiral in a coordinate system with a fixed EarthSun line. An Archimedian spiral leading back to the Sun is located between $30^{\circ} \mathrm{W} \div 80^{\circ} \mathrm{W}$ for a solar wind speed from $700 \mathrm{~km} / \mathrm{s}^{-1}$ to $300 \mathrm{~km} / \mathrm{s}^{-1}$, respectively. A priori, if the "associated" flare is situated inside or near this longitudinal interval, the protons have the highest probability to be registered near the Earth's orbit.
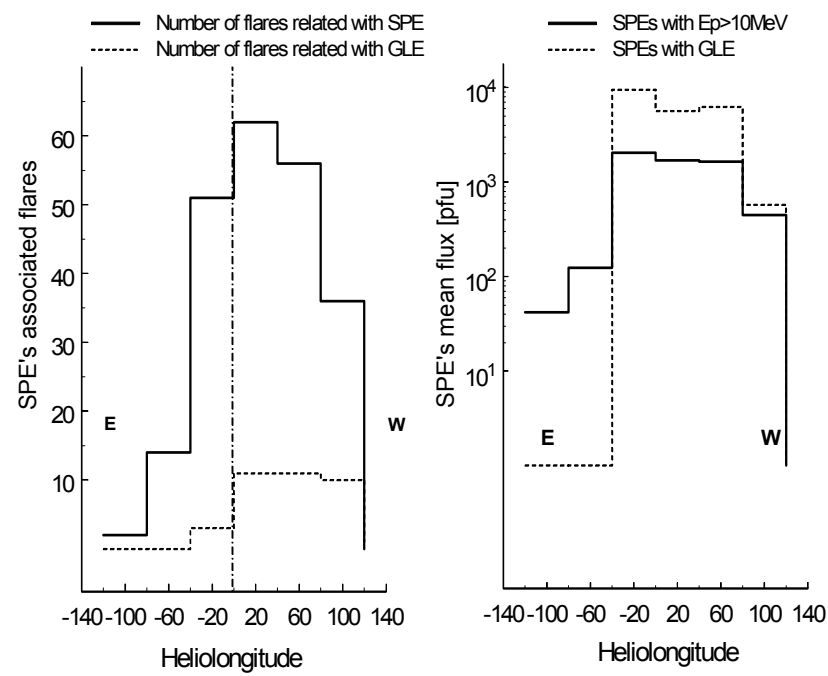

Fig. 6. Longitudinal distribution of the associated with SPEs flares and of the flares related to GLEs concerning the number of them (left panel) and the mean flux per $20^{\circ}$ longitudinal interval (right panel).

The longitudinal distribution of the associated SPE flares and the distribution of the flares connected with GLEs are presented in Fig. 6. The associated flares are more widely distributed from the east limb to $120^{\circ} \mathrm{W}$ and centered within the longitude interval $40 \mathrm{E} \div 80 \mathrm{~W}$, that is in accordance with previous works (Van Hollebeke et al., 1975; Sladkova and Bazilevskaya, 2000). In our case longitudinal distribution of the associated flare number, as well as of the mean flux of the corresponded SPEs, demonstrate that most of the SPEs originate from flares located western to $70^{\circ} \mathrm{W}$. It is interesting that only one of the GLEs has been registered from a flare located at the solar longitude $30^{\circ} \mathrm{E}$. All the others are created from flares located near the west limb. It is noteworthy that ten of the forty-two GLEs examined here are caused by "over the limb flares.

\subsection{Time delay}

It is known from $\gamma$-line observations that protons can be accelerated up to $10-30 \mathrm{MeV}$ energy during the rising phase of the flare and near the time of the flare maximum (Ramaty and Mandzhavidze, 1993; Chupp, 1987; 1996). There are evidences that additional prolonged acceleration takes place when the magnetic field in the flare region undergoes its restoration after the mass ejection and shock wave formation in the lower corona (e.g. Akimov et al., 1996). The exact time of the escaping of accelerated protons from the Sun into interplanetary space is unknown. It means that the zero value $\left(\mathrm{T}_{0}=\right.$ Tescaping $)$ of a time scale cannot be defined unambiguously. A suggestion was made that the time of the $\mathrm{H} \alpha$ flare onset (Table 1 , column 6 ) is taken as $\mathrm{T}_{0}$ for our time rule. Then, using the time maximum of the SPE as Tmax (Table 1, column 4), the time delay $\Delta \mathrm{T}$ between the proton event maximum and the onset time of the $\mathrm{H} \alpha$ "associated 

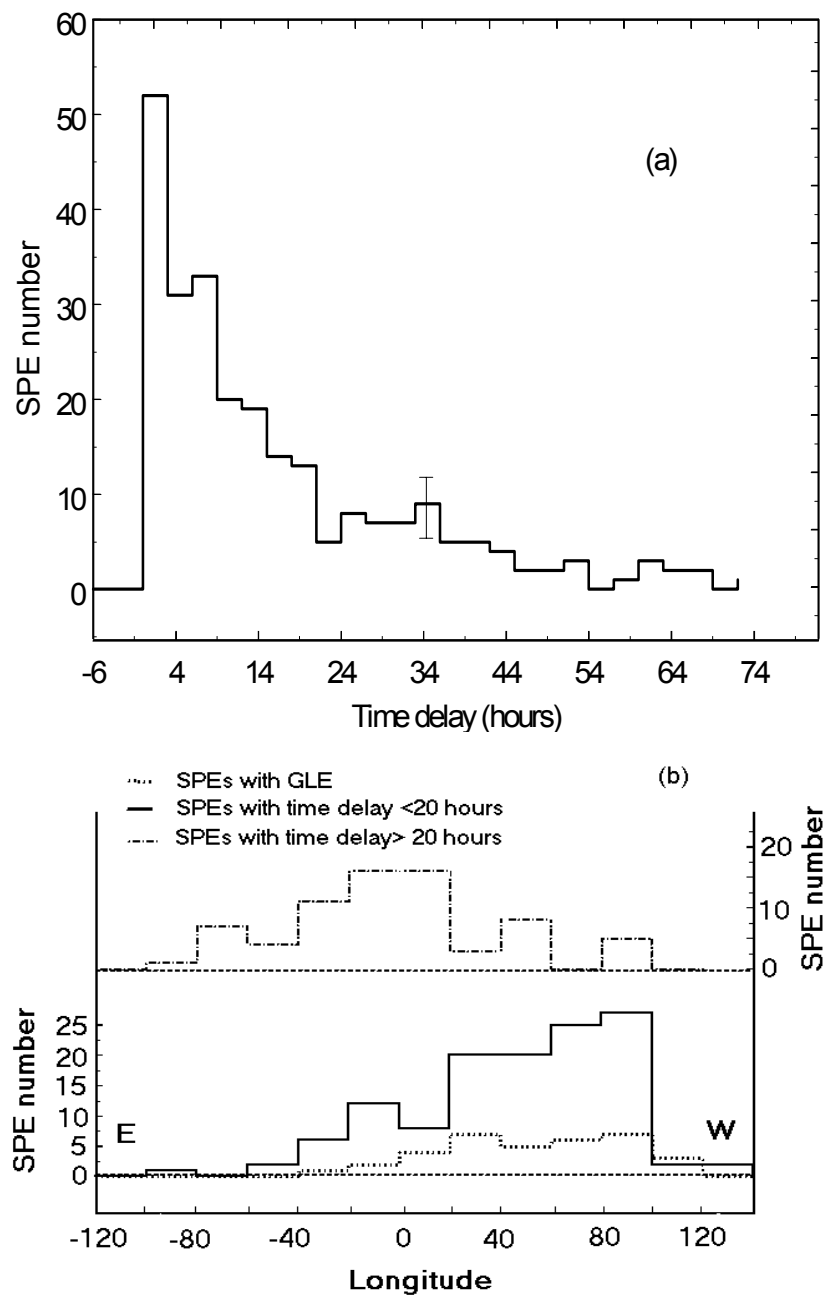

Fig. 7. (a) The SPEs number with respect the time delay between the proton event maximum and the onset time of the Ha "associated flares" is presented. (b) The longitudinal distribution of the SPEs in three cases (with GLE, with time delay $<20 \mathrm{~h}$ and with time delay $>20 \mathrm{~h}$ ) is also illustrated.

Table 2. SPEs number and Imean in latitudes $>20^{\circ}$ and $<20^{\circ}$.

\begin{tabular}{lll} 
Latitude & SPEs number & $\begin{array}{l}\text { Imean } \\
(\mathrm{pfu})\end{array}$ \\
\hline$>20^{\circ}$ & 59 & 3524 \\
$<20^{\circ}$ & 162 & 790 \\
\hline
\end{tabular}

flare" $\Delta \mathrm{T}=\mathrm{T}_{\max }-\mathrm{T}_{0}$ was calculated. Of course the choice of $\mathrm{T}_{0}$ is not physically correct, but it is acceptable, as the time resolution of our analysis is greater or equal to one hour.

\subsection{Associated solar flares}

A number of $231 \mathrm{H} \alpha / \mathrm{SXR}$ flares have been identified with respect to the solar proton events of our catalogue, named as "associated" flares of SPEs. From the rest of the SPEs some of them can possibly be connected with "over the limb" flares, in some cases the identification could not be done definitely, while some others are related to CMEs, not associated with flares.

Thus, a "butterfly" diagram-that means a time dependence of the solar latitudinal distribution of the "associated flares" is presented in Fig. 5. Monthly values of sunspot number are also given in this Figure, and the arrows indicate the start of each cycle. It is known that solar flares follow the "butterfly" diagram, by which every cycle starts with flares at high latitudes (greater than $35^{\circ}$, while it ends with flares at low latitudes. It is interesting to note, that in our case the latitudinal distribution of the associated flares seems to have a similar distribution to total flares and sunspot number. However, the sources of SPEs with large fluxes are often located at high latitudes greater than $20^{\circ}$ (Table 2). Apparently, the protons can propagate easily from relatively high latitudes to the ecliptic plane.

The result of our treatment on SPEs with respect to the calculated time delay $\Delta \mathrm{T}$ is demonstrated in Fig. 7a. One can see a very pronounced maximum in the time delay $\Delta \mathrm{T}$ from 3 to $9 \mathrm{~h}$, followed by a long lasting tail. SPEs can be separated into two groups depending on $\Delta \mathrm{T}$ values: the first one includes events with $\Delta \mathrm{T} \leq 20 \mathrm{~h}$ and the second one regarding events with $\Delta T \geq 20 \mathrm{~h}$. Longitudinal distributions of the "associated flares" in these two groups are depicted in Fig. 7b. The distribution of GLEs is also presented in the same figure. "Associated flares" in the first group obviously exhibit the same longitudinal distribution as those associated with GLEs, with most of them located in the Western Hemisphere.

This SPEs separation with respect to the time delay between the flare onset and the event maximum was found in accordance with the main properties of the proton's interplanetary transport. If an event has $\Delta \mathrm{T} \leq 20 \mathrm{~h}$, we may be sure that the registered event is "prompt", that means the proton transport is mainly caused by diffusion (Miroshnichenko, 2001). For the events of the second group an influence of shock waves and CMEs on the particle propagation and acceleration is noticeable. Sometimes the events with $\Delta \mathrm{T}$ between $20 \div 30 \mathrm{~h}$ are of "prompt" increases. In some cases the time of the peak maximum Tmax was coincided with a very fast shock arrival at $1 \mathrm{AU}$. Careful examination of the events with $\Delta \mathrm{T} \geq 30 \mathrm{~h}$ shows that these are shock-related events.

\subsection{Peak-size distributions}

The frequency distributions of our data set at the threshold proton energy of $10 \mathrm{MeV}$ and peak intensity $>10 \mathrm{pfu}$ represented by power law as $\mathrm{dN} / \mathrm{dI}=\mathrm{I}^{-v}$, where $\mathrm{N}$ is the number of events per flux interval and $\mathrm{I}$ is the mean particle flux in this interval at energy $>10 \mathrm{MeV}$, are presented in Fig. 8 . This figure includes differential frequency-size distributions of the peak value selected SPEs for three separate subsets. The first subset contains the total number of SPEs (upper panel), the second group contains the "prompt" SPEs with time delay $<20 \mathrm{~h}$ (middle panel) and the third one presents 
SPEs associated with GLEs (bottom panel). The first and second cases can be described by power law with exponent $\nu=-1.36 \pm 0.04$ and $\nu=-1.30 \pm 0.02$, respectively. The turnover near two bins $(30<\operatorname{Imax}<300 \mathrm{pfu})$ is seen in the peak-size distribution for the events with GLEs caused by threshold effects of the neutron monitors. The power law approximation outside the first bin $(30<\operatorname{Imax}<100 \mathrm{pfu})$ gives $\nu=1.12 \pm 0.16$. It is noticeable that a difference in the slopes between the differential distributions at $10 \mathrm{MeV}$ and $>500 \mathrm{MeV}$ (GLEs) has appeared. Hence, in our study the spectral indices vary from 1.12 to 1.36 . Such a difference in the slopes of solar proton events in different energy channels $(>10 \mathrm{MeV},>30 \mathrm{MeV},>60 \mathrm{MeV},>100 \mathrm{MeV},>500 \mathrm{MeV})$ for the time period 1970-1995 is also reported by Kurt et al. (2002). It indicates the existence of a slope dependence on the proton energy under consideration (Miroschnishenko et al., 2001).

Our results are consistent with spectral indices of solar proton events published earlier, as $1.15 \pm 0.1$ (Van Hollebeke, 1975), 1.45 \pm 0.15 (Belovsky and Ochelkov, 1979), $1.35 \pm 0.15$ (Kurt, 1990), $1.3 \pm 0.12$ (Gerontidou et al., 2002). In our case our results are based on the best statistics and give the verification of the spectral index that is very important for the models of flare energy release.

\section{Discussion and conclusions}

In this work the first attempt to accomplish an extended statistical analysis of solar proton events with energy $>10 \mathrm{MeV}$ and peak flux $>10$ pfu observed at 1 AU through January 1970 to December 2002 is performed. A catalogue of 253 events based upon satellites and ground level observations is created and presented. Solar proton event evolution steps, as time dependence over three solar cycles, longitudinal and latitudinal distributions of the parent flares and distribution of the time delay between the Ha flare onset and the SPE maximum are analyzed. The frequency peak flux distributions are also obtained.

Summing up the main results of this study we note that, together with appropriate results published since 1975, our findings provide new and important information about some features of the Sun's proton productivity and its relation to existing problems of particle acceleration at/near the Sun.

1. It is characteristic that the numbers of SPEs and SXR flares of importance $>\mathrm{M} 4$ are almost the same during the solar cycles 21 and 22. It is noted that one per six of these SXR flares is associated with an SPE. In the cycle 21 the number of SXR-flares of importance $>$ M4 is 478 and the number of SPEs is 78 , while during the solar cycle 22 the number of SXR-flares of importance $>$ M4 is 460 and the number of SPEs is 73 .

The occurrence rate of SPEs and SXR flares $>$ M4 in relation to other manifestations of solar activity seems to appear as a significant increase during the maximum of solar cycle 22. It means that solar activity with soft $\mathrm{x}$ -

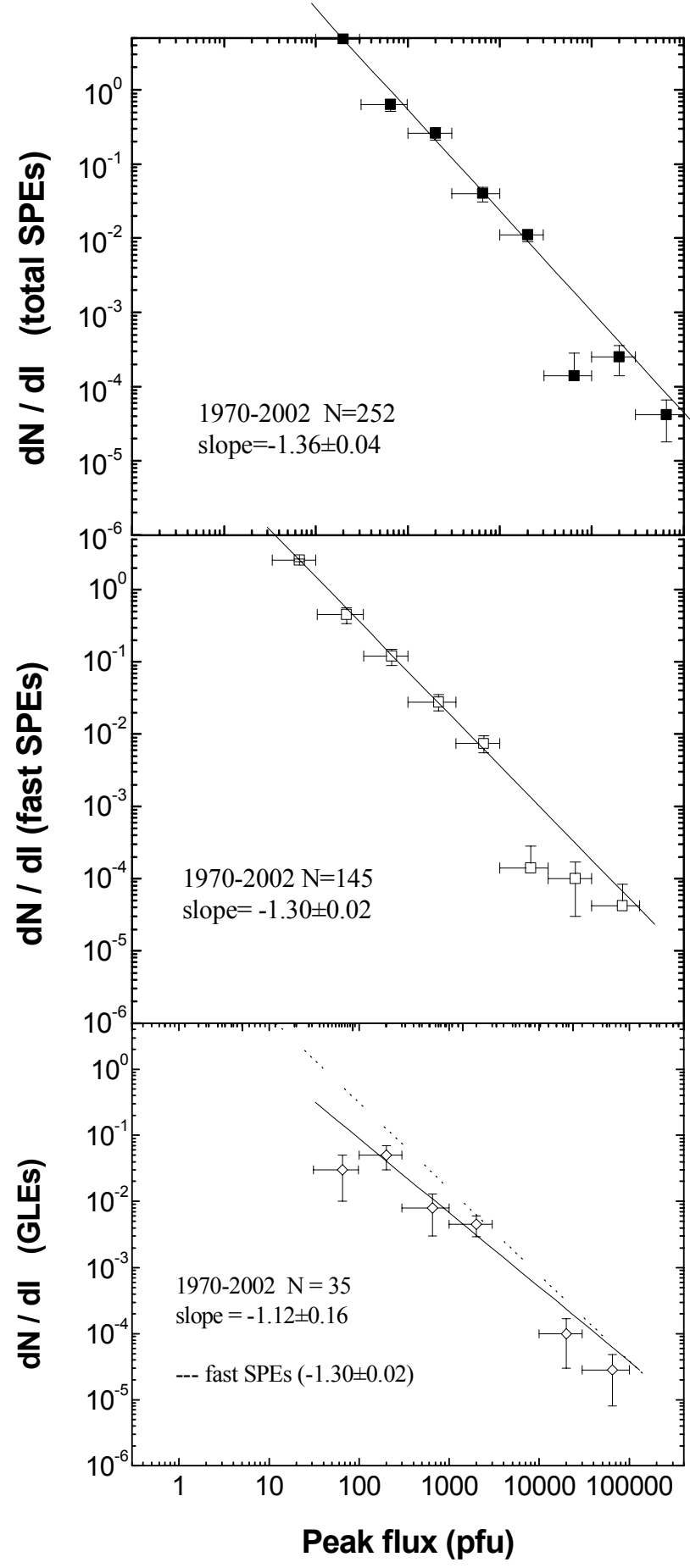

Fig. 8. Peak size distributions of the total number of SPEs with energies $\mathrm{E}>10 \mathrm{MeV}$ (upper panel), of the fast SPEs (middle panel) and of the SPEs connected with GLEs (lower panel) that appeared.

rays of importance $>\mathrm{M} 4$ have an ability to emit protons sufficient to be registered near the Earth's vicinity as SPEs, but not all of them are accompanied by a proton event. 
2. Dependence of solar latitude distribution of parent flares of SPEs on the solar cycle seems to have the same behavior as the sunspot number. Every cycle starts with flares at high latitudes and ends with flares at low latitudes. The sources of SPEs with large fluxes are often located at high latitudes greater than $20^{\circ}$. Protons can propagate easily from relatively high latitudes to the ecliptic plane. On the other hand, the longitudinal distribution of the associated flares of SPEs shows a preferable connecting region within $40^{\circ} \mathrm{E}-$ $80^{\circ} \mathrm{W}$, which is consistent with previous results (Sladkova and Basilevskaya, 2000). The most of the SPEs are created by flares, located at solar longitude around $70^{\circ} \mathrm{W}$. It is in agreement with the distribution of the GLE related flares.

3. The calculated time delay between the proton event maximum and the onset time of the $\mathrm{Ha}$ associated flare reveals a pronounced maximum of this time from 3 to $9 \mathrm{~h}$. This maximum is connected with fast events $(\Delta \mathrm{T}<20$ hours), where the proton transport is mainly caused by diffusion, while a long time delay is related to shock events.

4. The best power-law fit for the basic sample of 253 events is attained at a slope of $1.36 \pm 0.04$ over the entire range of proton intensities $10^{1}-10^{5} \mathrm{pfu}$. The difference in the slopes between differential size distributions at $>10 \mathrm{MeV}$ and $>500 \mathrm{MeV}$, obtained on the best statistic, indicates the existence of slope dependence on the proton energy. This result is consistent with other studies (Miroschnishenko et al., 2001; Gerontidou et al., 2002; Kurt et al, 2002).

5. The mechanisms responsible for proton acceleration and SPEs are widely discussed in the literature, with much controversy in particular over the role of flares. Our results not only confirm but give quantitative characteristics of the SPE relation to the flares that may be used for prediction of these events and radiation forecasting (Gabriel and Patrick, 2003).

Acknowledgements. This work is partly supported by the grants $70 / 4 / 6255$ and 70/4/6890 of the Ministry of Development of the General Secretariat of Research and Technology concerning GreekRussian Collaboration. Thanks are also due to the Special Research Account of Athens University for supporting this research. The authors are grateful to the anonymous referee for very valuable comments on this work.

Topical Editor R. Forsyth thanks D. Smart for his help in evaluating this paper.

\section{References}

Akimov, V. V., Ambroz, P., and Belov, A. V. et al.: Evidence for prolonged acceleration based on a detailed analysis of the longduration solar gamma-ray flare on 15 June, 1991, Solar Phys.,V. 166, 107-134, 1996.
Basilevskaya, G. A., Vashenyuk, E. V., and Ishkov, V. N. et al.: in Yu, I. Logachev (ed), Solar proton events Catalogue, WDC-B2, Moscow, 1983; 1986.

Belov A., Kurt, V., Gerontidou, M., and Mavromichalaki, H.: Statistical analysis of solar proton events in different energy channels, Proc. 27th ICRC 2001, 3465-3468, 2001.

Belovsky, M. H. and Ochelkov, Yu. P.: On some pecularities of generation of electromagnetic and corpuscular radiation in solar flares,Izvestia AN SSSR, Phys. Ser., 43, 4, 749-752, 1979.

Cane, H. V., Reames, D. V., and von Rosenvinge, T. T.: The role of interplanetary shocks in the longitude distribution of solar energetic particles, J. Geophys. Res., 93, A9, 9555-9567, 1988.

Chupp, E. L.: High energy particle production in solar flares (SEP, gamma-ray and neutron emissions),Physica Scripta, T18, 5-9, 1987.

Chupp, E. L.: Evolution of our understanding of solar flare particle acceleration:(1942-1995), in High Energy Solar Physics, eds.: R. Ramaty, N. Mandzhavidze, and X.-M. Hua, AIP Conference Proceedings, AIP: New York, 374, 3-31, 1996.

Cliver, E. W., Kahler, S. W., Shea, M. A., and Smart, D. F.: Injection onset of $\sim 2 \mathrm{GeV}$ protons, $\sim 1 \mathrm{MeV}$ electrons, and $\sim 100 \mathrm{keV}$ electrons in solar cosmic ray flares,Ap. J., 260, 362-370, 1982.

Cliver, E. W., Reames, D. V., Kahler, S. W., Cane, H. V.: Size distribution of solar energetic particle events, Proc. $22^{\text {nd }}$ ICRC, Dublin, 3, 25-28, 1991.

Crosby, N. B., Aschwanden, M. J., and Dennis, B. R.: Frequency distributions and correlations of solar X-ray flare parameters, Solar phys. 143, 275-299, 1993.

Dorman, L. I. and Miroshnichenko, L. I.: Solar cosmic rays, Moskow, Nauka (Fizmatgiz), English Edition for NASA by Indian national Scientific Documentation Center, Delhi, 1968.

Feynman, J., Spitale, G., Wang, J., and Gabriel, S.: Interplanetary proton fluence model, J. Geophys. Res., 98, A8, 13 281-13 294, 1993.

Forman, M. A., Ramaty, R., and Zweibel, E. G.: The acceleration and propagation of solar flare energetic particles, in: Physics of the Sun, ed: Sturrock P. A., Dordrecht: D. Reidel Publ.Co., Ch.II, 249-289, 1986.

Gabriel, S. B. and Feynman, J.: Power-law distribution for solar energetic proton events, Sol. Phys., 165, 337-346, 1996.

Gabriel, S. B. and Patrick, G. J.: Solar energetic particle events: phenomenology and prediction Space Sci. Rev. 107, 55-62, 2003.

Gerontidou , M., Vassilaki, A., Mavromichalaki, H., and Kurt, V.: Frequency distributions of solar proton events JASTP 64, 482490, 2002.

Goswami, J. N., Mc Guire, R. E., Reedy, R. C. et al.: Solar flare protons and alpha particles during the last three solar cycles,J. Geophys. Res., 93, A7, 7195-7205, 1988.

King J.H, Solar proton fluences for 1977-1983 Space Missions,J. Spacecraft and Rockets, 11, 6, 401-408, 1984.

Kurt V. G., Mavromichalaki, H., and Gerontidou, M.: Energy dependence of the solar proton events at 1 AU, Proc. "SOLMAG: magnetic Coupling of the Solar Atmosphere Euroconference and IAU Colloquium 188", 2002.

Kurt, V. G.: Electrons and X-ray emission of solar flares,in Basic Plasma Processes on the Sun, Eds: E. R. Priest and V. Krishan, 409-413, 1990.

Mason, G. M., Gloeckler, G., and Hovestadt, D.: Temporal variations of nucleonic abundances in solar flare energetic particle events. II. Evidence for large scale shock acceleration, Ap. J., 280, 902-916, 1984. 
McCracken, K. G.: The cosmic ray flare effect. I. Some new methods of analysis,J. Geophys. Res., 67, 2, 423-434, 1962.

Mendoza , B., Medenlez, R., Mirochnichenko, L. I., PerezEnriquez, R.: Frequency distributions of solar proton events, Proc 25th ICRC 1, 81-84, 1997.

Miroshnichenko, L. I.:in Solar Cosmic Rays, Kluwer Academic Publishers, 2001.

Miroshnichenko, L. I., Mendoza, B., and Perez-Enriquez, B.: Size distributions of the $>10 \mathrm{MeV}$ solar proton events, Solar Phys. 202, 151-171, 2001.

NOAA SEC Solar proton Events, Solar Geophysical Data, NOAA Boulder Co. 2002.

Ozguc, A. and Atac, T.: The 73- day periodicity of the flare index during the current solar cycle 22, Solar Phys. 150, 339, 1994.

Ramaty, R., Mandzhavidze, N., Kozlovsky, B., and Skibo, J. G.: Acceleration in solar flare: Interacting particles versus interplanetary particles, Adv. Space Res., 13, 9, 275-284, 1993.

Reames, D. V.: Particle acceleration at the sun and in the heliosphere, Space Sci. Rev. 90, 413-491, 1999.

Richter, A. K., Verigin, M. I., Kurt, V. G., et al.: The 3 January 1978 interplanetary shock event as observed by energetic particle, plasma and magnetic field devices on board of HELIOS1, HELIOS-2 and PROGNOZ-6,J. Geophys. Res., 50, 101-109, 1981.

Roelof, E. C.: Propagation of solar cosmic rays in the interplanetary magnetic field, in Lectures in High Energy Astrophysics, Eds: Hogelman and J.R.Wayland,NASA Spec. Publ., Sp 199.111$135,1969$.

Rusin, V. and Rybansky, M.: Coronal index of solar activity, Solar Phys. 207, 47, 2002.

Shea, M.A. and Smart, D.F, A summary of major solar proton events, Solar Phys.,127, 297-320, 1990.
Shea, M. A. and Smart, D. F.: A comparison of energetic solar proton events during the declining phase of four solar cycles (19-22) Adv. Space Res. 16, 9, 37-46, 1995.

Sladkova, A. I., Basilevskaya, G. A., Ishkov, V. N. et al.: Catalogue of solar proton events (ed) Y. Logachev, Moscow University Press 1990; 1998.

Sladkova, A. I. and Bazilevskaya, G. A.: On the origin of the relativistic protons observed in heliosphere after some solar flares, Adv. Space Res. 21, 559-562, 2000.

Stolpovsky, V. G., Kurt, V. G., and Logachev, Yu. I.: Catalogue solar particle events measured on board Spacecraft VENERA-13, 14. Preprint SINP MSU, No 34, Moscow, 1988.

Svestka, Z. and Simon, P.: Catalogue of solar particle events 19551969 Astrophys. and Space Sci. Library, 49 Reidel Publ. Co, Dordrecht Holland 1975.

Temmer, M. A., Veronig, A., Hanslmeier, W., and Otruba and Messerotti, M.: Statistical analysis of solar Ha flares Astron.\&Astrophys. 375, 1049-1061, 2001.

Valdes-Galicia, J. F. V., Moussas, X., and Quenby, J. J. et al.: Mean free paths and diffusion coefficients for energetic protons at small heliodistances calculated using HELIOS 1 and 2 data, Sol. Phys. V. 91, 399, 1984.

Van Hollebeke, M. A., MaSung, L. S., and McDonald, F. M.: The variation of solar proton energy spectra and size distribution with heliolongitude, Solar Phys., 41, 189-223, 1975.

Van Hollebeke, M. A., Wang, J. R., and McDonald, F. B.: A catalogue of solar cosmic ray events, X-661-74-27,NASA Goddard Space Flight Center, 1974.

Wibberenz, G., Kunow H., and Müller-Mellin R. et al.: Solar energetic and shock-accelerated particles observed between 1 and 4 AU by the Kiel electron telescope (KET) on board ULYSSES,J. Geophys. Res. Letters, 19, 12, 1279-1282, 1992. 